SAND2011-3282

Unlimited Release

Printed January 2011

\title{
TChem - A Software Toolkit for the Analysis of Complex Kinetic Models
}

Cosmin Safta, Habib Najm, Omar Knio

Prepared by

Sandia National Laboratories

Albuquerque, New Mexico 87185 and Livermore, California 94550

Sandia National Laboratories is a multi-program laboratory managed and operated by Sandia Corporation,

a wholly owned subsidiary of Lockheed Martin Corporation, for the U.S. Department of Energy's

National Nuclear Security Administration under contract DE-AC04-94AL85000.

Approved for public release; further dissemination unlimited. 

by Sandia Corporation.

NOTICE: This report was prepared as an account of work sponsored by an agency of the United States Government. Neither the United States Government, nor any agency thereof, nor any of their employees, nor any of their contractors, subcontractors, or their employees, make any warranty, express or implied, or assume any legal liability or responsibility for the accuracy, completeness, or usefulness of any information, apparatus, product, or process disclosed, or represent that its use would not infringe privately owned rights. Reference herein to any specific commercial product, process, or service by trade name, trademark, manufacturer, or otherwise, does not necessarily constitute or imply its endorsement, recommendation, or favoring by the United States Government, any agency thereof, or any of their contractors or subcontractors. The views and opinions expressed herein do not necessarily state or reflect those of the United States Government, any agency thereof, or any of their contractors.

Printed in the United States of America. This report has been reproduced directly from the best available copy.

Available to DOE and DOE contractors from

U.S. Department of Energy

Office of Scientific and Technical Information

P.O. Box 62

Oak Ridge, TN 37831

Telephone: $\quad$ (865) 576-8401

Facsimile: $\quad$ (865) 576-5728

E-Mail: reports@adonis.osti.gov

Online ordering: http://www.osti.gov/bridge

Available to the public from

U.S. Department of Commerce

National Technical Information Service

5285 Port Royal Rd

Springfield, VA 22161

Telephone: $\quad$ (800) 553-6847

Facsimile: (703) 605-6900

E-Mail: $\quad$ orders@ntis.fedworld.gov

Online ordering: http://www.ntis.gov/help/ordermethods.asp?loc=7-4-0\#online

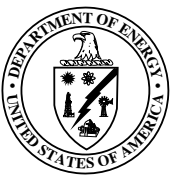




\title{
TChem - A Software Toolkit for the Analysis of Complex Kinetic Models
}

\author{
Cosmin Safta, Habib N. Najm \\ Sandia National Laboratories, Livermore, CA \\ \{csafta,hnnajm\}@sandia.gov \\ and \\ Omar Knio \\ Johns Hopkins University, Baltimore, MD \\ knio@jhu.edu
}

\begin{abstract}
The TChem toolkit is a software library that enables numerical simulations using complex chemistry and facilitates the analysis of detailed kinetic models. The toolkit provide capabilities for thermodynamic properties based on NASA polynomials and species production/consumption rates. It incorporates methods that can selectively modify reaction parameters for sensitivity analysis. The library contains several functions that provide analytically computed Jacobian matrices necessary for the efficient time advancement and analysis of detailed kinetic models.
\end{abstract}




\section{Acknowledgment}

This work was supported by the US Department of Energy (DOE), Office of Basic Energy Sciences (BES) Division of Chemical Sciences, Geosciences, and Biosciences. Sandia National Laboratories is a

multiprogram laboratory operated by Sandia Corporation, a Lockheed Martin Company, for the United States Department of Energy under contract DE-AC04-94-AL85000. 


\section{Contents}

1 Introduction $r$

2 Thermodynamic properties, equation of state, and mixture formulae 11

3 Reaction-rate expressions $r$

4 Jacobian Matrices $\quad 17$

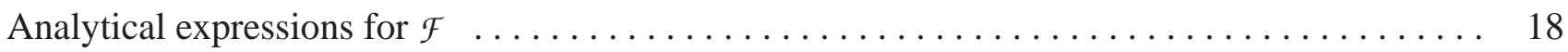

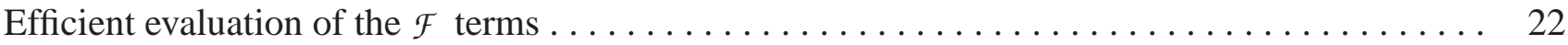

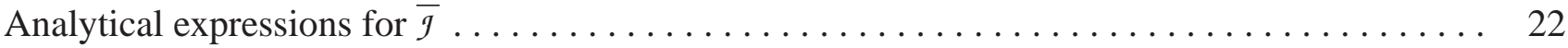

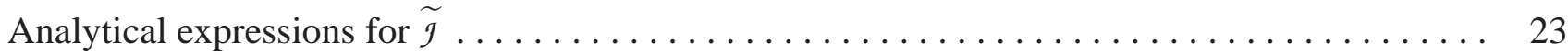

5 Examples $\quad 25$

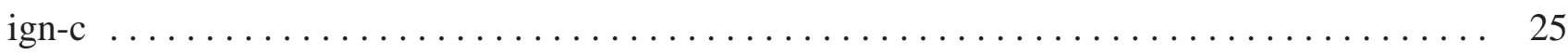

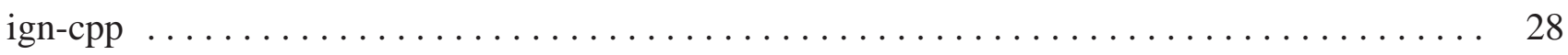

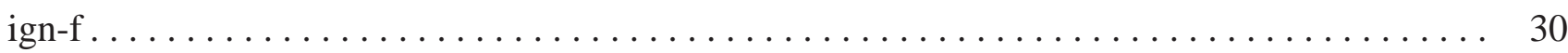

$\begin{array}{llr}6 & \text { Library Functions } & 33\end{array}$

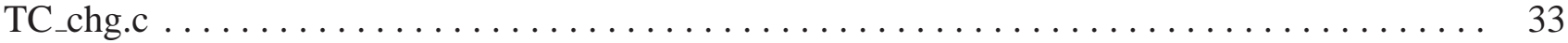

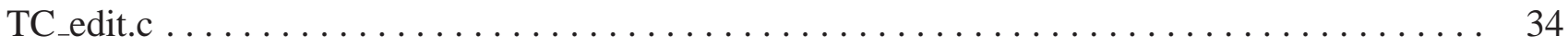

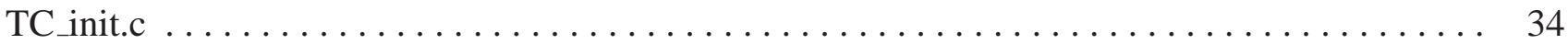

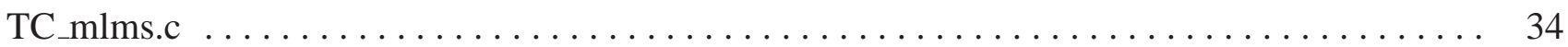

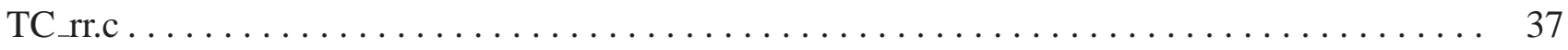

TC 


\section{Nomenclature}

Non-dimensional values

References 


\section{List of Figures}

5.1 Left Frame: variation of the ignition delay times with the equivalence ratio $(\Phi)$ for isooctane/air mixtures at various pressures and initial temperature of 1000K. Right Frame: Variation of ignition delay time with initial temperature for a stoichiometric iso-octane/mixtures at various pressures. The kinetic model involves 874 species and 3796 reactions [5] . . . . 27

5.2 Left Frame: Variation of the ignition delay times with the initial mixture temperature for $100 \mathrm{ON}, 80 \mathrm{ON}$ and $60 \mathrm{ON}$ at $15 \mathrm{~atm}$ and $45 \mathrm{~atm}$. Right Frame: Variation of ignition delay time for $50 \mathrm{ON}$ at $15 \mathrm{~atm}, 30 \mathrm{~atm}$, and $45 \mathrm{~atm}$. The kinetic model involves 1034 species

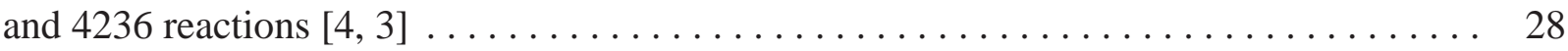

5.3 Mass fraction profiles during the constant pressure ignition of a stoichimetric methane-air mixture at 1atm. Methane combustion is modeled using the GRI-Mech v3.0 kinetic model (53 species, 325 reactions) [7]. 
This page intentionally left blank. 


\section{Chapter 1}

\section{Introduction}

Consider the system of stiff ordinary differential equations (ODE's) that describe the evolution of a homogenous mixture of $N_{\text {spec }}$ species in an open container

$$
\begin{aligned}
& \frac{\partial T}{\partial t}=-\frac{1}{\rho c_{p}} \sum_{k=1}^{N_{\mathrm{spec}}} h_{k} \dot{\omega}_{k} \\
& \frac{\partial Y_{k}}{\partial t}=\frac{\dot{\omega}_{k}}{\rho} \quad k=1 \ldots N_{\mathrm{spec}}
\end{aligned}
$$

where $\rho$ is the density, $T$ the temperature, $Y_{k}$ the mass fraction of species $k, c_{p}$ and $c_{p_{k}}$ the specific heat of the mixture and species $k$, respectively and $\dot{\omega}_{k}$ the reaction rate of species $k$. In some cases, the ODE for select species (usually the most abundant in the mixture) is replaced by an equation enforcing mass conservation. For example

$$
Y_{L}=1-\sum_{k=1, k \neq L}^{N_{\text {spec }}} Y_{k}
$$

can substitute the ODE for species $L$ in (1.1).

The system of equations (1.1) can aid in the design of kinetic models for increasingly complex fuels. It allows the computation of ignition delay times and mixture compositions that can be compared against experimental results from shock tube experiments. This system is a canonical configuration which enables the study of chemical reaction pathways and testing of algorithms for reducing detailed kinetic models for use in more complex configurations.

TChem is as an open source software library that facilitates the analysis of complex kinetic models and provides tools for incorporating these models in combustion simulations. The example codes, written in $\mathrm{C}, \mathrm{C}++$, and Fortran, that are part of the library provide model solvers for the system of ODE 1.1. While the library is standalone, the example applications require the open source libraries CVODE ${ }^{1}$ (for the $\mathrm{C}$ and C++ models) and DVODE ${ }^{2}$ (for the Fortran model).

This report is organized as follows. Chapter 2 contains expressions for the thermodynamic properties and chemical composition formulae implemented in the library. Chapter 3 provides an overview of the reaction rate expressions. Chapter 4 describes the derivation and calculation of the Jacobian matrices.

\footnotetext{
${ }^{1}$ https://computation.llnl.gov/casc/sundials

${ }^{2}$ https: //computation.llnl.gov/casc/software.html
} 
Chapter 5 describes $\mathrm{C}, \mathrm{C}++$ and Fortran example codes that use TChem. The user interface for all functions is presented in Chapter 6. 


\section{Chapter 2}

\section{Thermodynamic properties, equation of state, and mixture formulae}

The heat capacity at constant pressure $\left(C_{p}\right)$, molar enthalpy $(H)$, and entropy $(S)$ for a mixture are given by :

$$
C_{p}=\sum_{k=1}^{N_{\text {spec }}} X_{k} C_{p_{k}}, \quad H=\sum_{k=1}^{N_{\text {spec }}} X_{k} H_{k}, \quad S=\sum_{k=1}^{N_{\text {spec }}} X_{k} S_{k}
$$

where subscript $k$ stands for species " $\mathrm{k}$ " and $N_{\text {spec }}$ is the number of species in the mixture. The standardstate thermodinamic properties for a thermally perfect gas are computed based on NASA polynomials [6]

$$
\begin{aligned}
& \frac{C_{p_{k}}}{\mathfrak{R}}=a_{0, k}+T\left(a_{1, k}+T\left(a_{2, k}+T\left(a_{3, k}+a_{4, k} T\right)\right)\right), \\
& \frac{H_{k}}{\mathfrak{R}}=T\left(a_{0, k}+T\left(\frac{a_{1, k}}{2}+T\left(\frac{a_{2, k}}{3}+T\left(\frac{a_{3, k}}{4}+\frac{a_{4, k}}{5} T\right)\right)\right)\right)+a_{5, k}, \\
& \frac{S_{k}}{\Re}=a_{0, k} \ln T+T\left(a_{1, k}+T\left(\frac{a_{2, k}}{2}+T\left(\frac{a_{3, k}}{3}+\frac{a_{4, k}}{4} T\right)\right)\right)+a_{6, k},
\end{aligned}
$$

Here $X_{k}$ the mole fraction of species $k$, and $\Re$ the universal gas constant. The specific thermodynamic properties in mass units are optained by dividing the above expressions by the molecular weights:

$$
c_{p_{k}}=C_{p_{k}} / W_{k}, \quad h_{k}=H_{k} / W_{k}, \quad s_{k}=S_{k} / W_{k}
$$

and the mixture properties computed as

$$
c_{p}=\sum Y_{k} c_{p_{k}}, \quad h=\sum Y_{k} h_{k}, \quad s=\sum Y_{k} s_{k}
$$

where $W_{k}$ and $Y_{k}$ are the molecular weight and the mass fraction of species $k$, respectively.

The ideal gas equation of state is used throught the library,

$$
P=\rho \frac{\mathfrak{R}}{\bar{W}} T=\left(\sum_{k=1}^{N_{\text {spec }}} \mathfrak{X}_{k}\right) \Re T
$$

where $P$ is the thermodynamic pressure, $\bar{W}$ is the molecular weight of the mixture, $T$ is the temperature, and $\mathfrak{X}_{k}$ is the molar concentration of species $k . \bar{W}$ is computed as

$$
\bar{W}=\left(\sum_{k=1}^{N_{\text {spec }}} \frac{Y_{k}}{W_{k}}\right)^{-1}=\sum_{k=1}^{N_{\text {spec }}} X_{k} W_{k} .
$$


Mass and mole fractions can be computed from each other as

$$
X_{k}=Y_{k} \bar{W} / W_{k}, \quad Y_{k}=X_{k} W_{k} / \bar{W}
$$

while the molar concentration is given by $\mathfrak{X}_{k}=\rho Y_{k} / W_{k}$.

The units for these properties are given in Section 7. Functions that perform calculations described in this section are contained in files TC_mlms.c and TC_thermo.c and are described in Section 6. 


\section{Chapter 3}

\section{Reaction-rate expressions}

The reaction rate of species $k$ in mass units is written as

$$
\dot{\omega}_{k}=W_{k} \sum_{i=1}^{N_{\mathrm{reac}}} \mathrm{v}_{k i} q_{i}, \quad \mathrm{v}_{k i}=\mathrm{v}_{k i}^{\prime \prime}-\mathrm{v}_{k i}^{\prime}
$$

where $N_{\text {reac }}$ is the number of reactions and $v_{k i}^{\prime}$ and $v_{k i}^{\prime \prime}$ are the stoichiometric coefficients of species $k$ in reaction $i$ for the reactant and product side of the reaction, respectively. The rate-of-progress of reaction $i$ is $q_{i}=C_{i} \mathcal{R}_{i}$, with

$$
C_{i}= \begin{cases}1 & \text { basic reaction } \\ \mathfrak{X}_{i} & 3^{\text {rd }} \text { body enhanced, no pressure dependence } \\ \frac{P_{r i}}{1+P_{r i}} F_{i} & \text { unimolecular/recombination fall-off reactions } \\ \frac{1}{1+P_{r i}} F_{i} & \text { chemically activated bimolecular reactions }\end{cases}
$$

and $\mathcal{R}_{i}=k_{f_{i}} \prod_{j=1}^{N_{\text {spec }}} \mathfrak{X}_{j}^{v_{j i}^{\prime}}-k_{r i} \prod_{j=1}^{N_{\text {spec }}} \mathfrak{X}_{j}^{v_{j i}^{\prime \prime}}$. The above expressions are detailed below :

- Forward rate constant has an Arrhenius expression, $k_{f_{i}}=A_{i} T^{\beta_{i}} \exp \left(-\frac{E_{a i}}{\Re T}\right)$, where $A_{i}, \beta_{i}$, and $E_{a i}$ are the pre-exponential factor, temperature exponent, and activation energy, respectively, for reaction $i$.

- Reverse rate constant $k_{r i}$. For reactions with reverse Arrhenius parameters specified, $k_{r i}$ is computed similar to $k_{f_{i}}$. If the reverse Arrhenius parameters are not specified, $k_{r i}$ is computed as $k_{r i}=k_{f_{i}} / K_{c i}$, where

$$
\begin{aligned}
& K_{c i}=\left(\frac{p_{a t m}}{\mathfrak{R} T}\right)^{\sum_{k=1}^{N_{\mathrm{spec}}} v_{k i}} K_{p_{i}}=\left(\frac{p_{\text {atm }}}{\mathfrak{R} T}\right)^{\sum_{k=1}^{N_{\mathrm{spec}}} v_{k i}} \exp \left(\sum_{k=1}^{N_{\mathrm{spec}}} \mathrm{v}_{k i}\left(\frac{s_{k}}{R_{k}}-\frac{h_{k}}{R_{k} T}\right)\right) \\
& =\left(\frac{p_{a t m}}{\mathfrak{R}}\right)^{\sum_{k=1}^{N_{\text {spec }}} v_{k i}} \exp \left(\sum_{k=1}^{N_{\text {spec }}} v_{k i}\left(-\ln T+\frac{s_{k}}{R_{k}}-\frac{h_{k}}{R_{k} T}\right)\right) \\
& =\left(\frac{p_{a t m}}{\mathfrak{R}}\right)^{\sum_{k=1}^{N_{\mathrm{spec}}} v_{k i}} \exp \left(\sum_{k=1}^{N_{\text {spec }}} \mathrm{v}_{k i} g_{k}\right) \text {. }
\end{aligned}
$$


Based on the polynomial expressions in (2.3) and (2.4), $g_{k}$ 's are computed as

$$
\begin{aligned}
g_{k} & =-\ln T+\frac{s_{k}}{R_{k}}-\frac{h_{k}}{R_{k} T} \\
& =a_{6, k}-a_{0, k}+\left(a_{0, k}-1\right) \ln T+T\left(\frac{a_{1, k}}{2}+T\left(\frac{a_{2, k}}{6}+T\left(\frac{a_{3, k}}{12}+\frac{a_{4, k}}{20} T\right)\right)\right)-\frac{a_{5, k}}{T}
\end{aligned}
$$

Note: If a reaction is irreversible, $k_{r}=0$.

- Concentration of the "third-body", $\mathfrak{X}_{i}=\sum_{j=1}^{N_{\text {spec }}} \alpha_{i j} \mathfrak{X}_{j}$, where $\alpha_{i j}$ is the efficiency of species $j$ in reaction $i$ and $\mathfrak{X}_{j}$ is the concentration of species $j . \alpha_{i j}$ coefficients are set to 1 unless specified in the kinetic model description.

- Reduced pressure $P_{r i}$. If expression " $(+\mathrm{M})$ " is used to describe a reaction, then $P_{r i}=\frac{k_{0 i}}{k_{\infty i}} \mathfrak{X}_{i}$. For reactions that contain expressions like " $\left(+\Upsilon_{m}\right)$ " $\left(\Upsilon_{m}\right.$ is the name of species $\left.m\right), P_{r i}=\frac{k_{0 i}}{k_{\infty i}} \mathfrak{X}_{m}$.

For unimolecular/recombination fall-off reactions the Arrhenius parameters for the high-pressure limit rate constant $k_{\infty}$ are given on the reaction line, while the parameters for the low-pressure limit rate constant $k_{0}$ are given on the auxiliary reaction line that contains the keyword "LOW". For chemically activated bimolecular reactions the parameters for $k_{0}$ are given on the reaction line while the parameters for $k_{\infty}$ are given on the auxiliary reaction line that contains the keyword "HIGH".

$$
F_{i}= \begin{cases}1 & \text { Lindemann reaction } \\ F_{c e n t}^{1 /\left(1+(A / B)^{2}\right)} & \text { Troe reaction } \\ d T^{e}\left(a \exp \left(-\frac{b}{T}\right)+\exp \left(-\frac{T}{c}\right)\right)^{X} & \text { SRI reaction }\end{cases}
$$

1. For the Troe form, $F_{c e n t}, A$, and $B$ are

$$
\begin{aligned}
F_{\text {cent }} & =(1-a) \exp \left(-\frac{T}{T^{* * *}}\right)+a \exp \left(-\frac{T}{T^{*}}\right)+\exp \left(-\frac{T^{* *}}{T}\right) \\
A & =\log _{10} P_{r i}-0.67 \log _{10} F_{c e n t}-0.4 \\
B & =0.806-1.1762 \log _{10} F_{c e n t}-0.14 \log _{10} P_{r i}
\end{aligned}
$$

Parameters $a, T^{* * *}, T^{*}$, and $T^{* *}$ are provided (in this order) in the kinetic model description for each Troe-type reaction. If $T^{* *}$ is ommited, only the first two terms are used to compute $F_{\text {cent }}$.

2. For the SRI form exponent $X$ is computed as $X=\left(1+\left(\log _{10} P_{r i}\right)^{2}\right)^{-1}$. Parameters $a, b, c, d$, and $e$ are provided in the kinetic model description for each SRI-type reaction. If $d$ and $e$ are ommited, these parameters are set to $d=1$ and $e=0$.

\section{Note on units for reaction rates}

In most cases, the kinetic models input files contain parameters that are based on calories, cm, moles, 
kelvin, seconds. The mixture temperature and species molar concentrations are necessary to compute the reaction rate. Molar concentrations are computed as

$$
\mathfrak{X}_{k}=\rho \frac{Y_{k}}{W_{k}} \quad\left[\frac{k m o l}{m^{3}}\right]
$$

The molar concentrations are then multiplied by $10^{-3}$ to convert them to $\left[\frac{\mathrm{mol}}{\mathrm{cm}^{3}}\right]$. The molar reaction rates computed based on these values are in $\left[\frac{\mathrm{mol}}{\mathrm{cm}^{3} \cdot \mathrm{s}}\right]$. These molar reaction rates are multiplied by $10^{3}$ and by the molecular weight of each species $\left[\frac{\mathrm{kg}}{\mathrm{kmol}}\right]$ to obtain the mass reaction rates in $\left[\frac{\mathrm{kg}}{\mathrm{m}^{3} \cdot \mathrm{s}}\right]$. 
This page intentionally left blank. 


\section{Chapter 4}

\section{Jacobian Matrices}

Efficient integration and accurate analysis of the stiff system of ODE's (1.1) requires the Jacobian matrix of the rhs vector. In this chapter we will derive the components of the Jacobian matrices both for systems which includes equations for all species as well as for systems of ODE's with one eqauation being replaced by the mass conservation equation (1.2).

Let

$$
\begin{aligned}
& \bar{\Phi}=\left\{P, T, Y_{1}, Y_{2}, \ldots, Y_{N_{\text {spec }}}\right\}^{T} \text { and } \\
& \widetilde{\Phi}=\left\{P, T, Y_{1}, \ldots, Y_{L-1}, Y_{L+1}, \ldots, Y_{N_{\text {spec }}}\right\}^{T}
\end{aligned}
$$

denote the set of variables for the full and reduced systems of ODE's, respectively. Altough we included pressure as an independent variable, we restrict our attention to open containers for which the thermodynamic pressure, $P$, is constant. Then (1.1) can be written in compact form as

$$
\begin{aligned}
& \frac{\partial \bar{\Phi}}{\partial t}=\bar{f}=\left\{S_{P}, S_{T}, S_{Y_{1}}, S_{Y_{2}}, \ldots, S_{Y_{N_{\mathrm{spec}}}}\right\}^{T} \text { and } \\
& \frac{\partial \widetilde{\Phi}}{\partial t}=\tilde{f}=\left\{S_{P}, S_{T}, S_{Y_{1}}, \ldots, S_{Y_{L-1}}, S_{Y_{L+1}}, \ldots, S_{Y_{N_{\mathrm{spec}}}}\right\}^{T},
\end{aligned}
$$

where $S_{P} \equiv 0, S_{T}=-\frac{1}{\rho c_{p}} \sum_{k=1}^{N_{\text {spec }}} h_{k} \dot{\omega}_{k}$, and $S_{Y_{k}}=\dot{\omega}_{k} / \rho$.

Let $\bar{g}$ and $\widetilde{g}$ be the Jacobian matrices corresponding to $\bar{f}$ and $\widetilde{f}$, respectively. In order to facilitate the derivation of partial derivatives $\partial \bar{f}_{i} / \partial \bar{\Phi}_{j}$ and $\partial \widetilde{f}_{i} / \partial \widetilde{\Phi}_{j}$ that are the elements of these matrices, it is useful to add density to the state vector. Let $\Phi$ be the extended state vector:

$$
\Phi=\left\{\rho, P, T, Y_{1}, Y_{2}, \ldots, Y_{N_{\text {spec }}}\right\}^{T},
$$

The rhs term corresponding to the time advance of $\Phi$ is

$$
f(\Phi)=\left\{S_{\rho}, S_{P},-\frac{1}{\rho c_{p}} \sum_{k=1}^{N_{\mathrm{spec}}} h_{k} \dot{\omega}_{k}, \frac{\dot{\omega}_{1}}{\rho}, \frac{\dot{\omega}_{2}}{\rho}, \cdots, \frac{\dot{\omega}_{N_{\mathrm{spec}}}}{\rho}\right\}^{T},
$$

with $S_{\rho}=-\bar{W} \sum_{k=1}^{N_{\text {spec }}} \frac{\dot{\omega}_{k}}{W_{k}}+\frac{1}{c_{p} T} \sum_{k=1}^{N_{\text {spec }}} h_{k} \dot{\omega}_{k}$. 


$$
\begin{aligned}
& \frac{\partial \bar{f}_{u}}{\partial v}=\frac{\partial f_{u}}{\partial v}+\frac{\partial f_{u}}{\partial \rho} \frac{\partial \rho}{\partial v} \\
& \frac{\partial \tilde{f}_{u}}{\partial v}=\frac{\partial f_{u}}{\partial v}+\frac{\partial f_{u}}{\partial \rho} \frac{\partial \rho}{\partial v}+\frac{\partial f_{u}}{\partial Y_{L}} \frac{\partial Y_{L}}{\partial v}
\end{aligned}
$$

Note that each component $u$ of $\widetilde{\Phi}$ is also a component of $\bar{\Phi}$ and $\Phi$, and the corresponding rhs terms are the same

$$
\widetilde{f}_{u}(\widetilde{\Phi})=\bar{f}_{u}(\bar{\Phi})=f_{u}(\Phi)
$$

In terms of actual components

$$
\begin{aligned}
\overline{\mathcal{g}}_{i, j}=\mathcal{F}_{i+1, j+1}+\mathcal{F}_{i+1,1} \frac{\partial \rho}{\partial \bar{\Phi}_{j}} & i, j=1,2, \ldots, N_{\text {spec }}+2 \\
\widetilde{\mathcal{g}}_{i, j}=\mathcal{F}_{i+i_{s}, j+j_{s}}+\mathcal{F}_{i+i_{s}, 1} \frac{\partial \rho}{\partial \widetilde{\Phi}_{j}}+\mathcal{F}_{i+i_{s}, L+3} \frac{\partial Y_{L}}{\partial \widetilde{\Phi}_{j}} & i, j=1,2, \ldots, N_{\text {spec }}+1 .
\end{aligned}
$$

where

$$
i_{s}, j_{s}= \begin{cases}1 & i, j+1<3+L \\ 2 & \text { otherwise }\end{cases}
$$

\section{Analytical expressions for $\mathcal{F}$}

Analytical for expressions for the matrix components are provided line by line below. Note that, in the expressions below, whenever the summation limits are omitted, the sums are over all $N_{\text {spec }}$ species.

- Line \#1: Density equation. Density is not currently advanced through an equation for the time being. Since $\mathcal{F}_{1 j}=\frac{\partial f_{1}}{\partial \Phi_{j}}$ are not needed, all components on the first line of $\mathcal{F}$ are set to 0 .

- Line \#2: Pressure equation. The source term for the pressure equation is assumed constant: $f_{2}=$ $\bar{f}_{2}=$ const.

$$
\frac{\partial f_{2}}{\partial \Phi_{j}} \equiv 0 \text { for all } \mathrm{j} \Rightarrow \mathcal{F}_{2, j} \equiv 0
$$

- Line \#3: Temperature equation: $\mathcal{F}_{3, j}=\frac{\partial f_{3}}{\partial \Phi_{j}}$, where $f_{3}=-\frac{1}{\rho c_{p}} \sum_{k=1}^{N_{\text {spec }}} h_{k} \dot{\omega}_{k}$. Here

$$
c_{p}=\sum_{k=1}^{N_{\text {spec }}} Y_{k} c_{p_{k}}(T) \text { and } \dot{\omega}_{k}=\dot{\omega}_{k}\left(T, \mathfrak{X}_{1}, \mathfrak{X}_{2}, \ldots, \mathfrak{X}_{N_{\text {spec }}}\right) .
$$


$\mathfrak{X}_{k}$ is the molar concentration of species $k$

$$
\mathfrak{X}_{k}=\frac{\rho Y_{k}}{W_{k}} .
$$

The individual components of $\mathcal{F}_{3, *}$ are computed as

$$
\begin{aligned}
\mathcal{F}_{3,1} & =\frac{\partial f_{3}}{\partial \rho}=\frac{1}{\rho^{2} c_{p}} \sum h_{k} \dot{\omega}_{k}-\frac{1}{\rho c_{p}} \sum h_{k} \frac{\partial \dot{\omega}_{k}}{\partial \rho}=\frac{1}{\rho c_{p}} \sum h_{k}\left(\frac{\dot{\omega}_{k}}{\rho}-\frac{\partial \dot{\omega}_{k}}{\partial \rho}\right) \\
\mathcal{F}_{3,2} & =0 \\
\mathcal{F}_{3,3} & =\frac{\partial f_{3}}{\partial T}=\frac{1}{\rho c_{p}^{2}} \frac{\partial c_{p}}{\partial T} \sum h_{k} \dot{\omega}_{k}-\frac{1}{\rho c_{p}} \sum c_{p_{k}} \dot{\omega}_{k}-\frac{1}{\rho c_{p}} \sum h_{k} \frac{\partial \dot{\omega}_{k}}{\partial T} \\
\mathcal{F}_{3,3+j} & =\frac{\partial f_{3}}{\partial Y_{j}}=\frac{1}{\rho c_{p}^{2}} c_{p_{j}} \sum h_{k} \dot{\omega}_{k}-\frac{1}{\rho c_{p}} \sum h_{k} \frac{\partial \dot{\omega}_{k}}{\partial Y_{j}}, j=1,2, \ldots, N_{\text {spec }}
\end{aligned}
$$

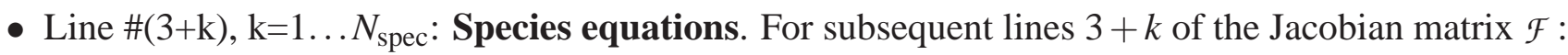

$$
\begin{aligned}
\mathcal{F}_{3+k, 1} & =\frac{\partial f_{3+k}}{\partial \rho}=\frac{\partial\left(\dot{\omega}_{k} / \rho\right)}{\partial \rho}=\frac{1}{\rho}\left(\frac{\partial \dot{\omega}_{k}}{\partial \rho}-\frac{\dot{\omega}_{k}}{\rho}\right) \\
\mathcal{F}_{3+k, 2} & =\frac{\partial\left(\dot{\omega}_{k} / \rho\right)}{\partial P} \equiv 0 \\
\mathcal{F}_{3+k, 3} & =\frac{\partial\left(\dot{\omega}_{k} / \rho\right)}{\partial T}=\frac{1}{\rho} \frac{\partial \dot{\omega}_{k}}{\partial T} \\
\mathcal{F}_{3+k, 3+j} & =\frac{\partial\left(\dot{\omega}_{k} / \rho\right)}{\partial Y_{j}}=\frac{1}{\rho} \frac{\partial \dot{\omega}_{k}}{\partial Y_{j}}, j, k=1,2, \ldots, N_{\text {spec }}
\end{aligned}
$$

The values for heat capacities and their derivaties are computed based on the NASA polynomial fits as

$$
\begin{aligned}
& \frac{\partial c_{p_{k}}}{\partial T}=R_{k}\left(a_{1, k}+T\left(2 a_{2, k}+T\left(3 a_{3, k}+4 a_{4, k} T\right)\right)\right), \\
& \frac{\partial c_{p}}{\partial T}=\sum Y_{k} \frac{\partial c_{p_{k}}}{\partial T}, \quad \frac{\partial c_{p}}{\partial Y_{j}}=c_{p_{j}}
\end{aligned}
$$

The partial derivatives of the species reaction rates, $\dot{\omega}_{k}\left(T, \mathfrak{X}_{1}, \mathfrak{X}_{2}, \ldots\right)$, with respect to various independend variables are computed as

$$
\begin{aligned}
\left.\frac{\partial \dot{\omega}_{k}}{\partial \rho}\right|_{T, Y_{s}} & =\sum_{l=1}^{N_{\text {spec }}} \frac{\partial \dot{\omega}_{k}}{\partial \mathfrak{X}_{l}} \frac{\partial \mathfrak{X}_{l}}{\partial \rho}+\frac{\partial \dot{\omega}_{k}}{\partial T} \frac{\partial T^{f}}{\partial \rho}+\frac{\partial \dot{\omega}_{k}}{\partial \rho} \frac{\partial \rho}{\partial \rho}=\sum_{l=1}^{N_{\text {spec }}} \frac{Y_{l}}{W_{l}} \frac{\partial \dot{\omega}_{k}}{\partial \mathfrak{X}_{l}} \\
\left.\frac{\partial \dot{\omega}_{k}}{\partial Y_{j}}\right|_{\rho, T, Y_{s \neq j}} & =\sum_{l=1}^{N_{\text {spec }}} \frac{\partial \dot{\omega}_{k}}{\partial \mathfrak{X}_{l}} \frac{\partial \mathfrak{X}_{l}}{\partial Y_{j}}+\frac{\partial \dot{\omega}_{k}}{\partial T} \frac{\partial T^{A}}{\partial Y_{j}}+\frac{\partial \dot{\omega}_{k}}{\partial \rho} \frac{\partial \rho^{\prime}}{\partial Y_{j}}=\frac{\rho}{W_{j}} \frac{\partial \dot{\omega}_{k}}{\partial \mathfrak{X}_{j}}
\end{aligned}
$$

The steps for the calculation of $\frac{\partial \dot{\omega}_{k}}{\partial T}$ and $\frac{\partial \dot{\omega}_{k}}{\partial \mathfrak{X}_{l}}$ are itemized below 
- Derivatives of reaction rate

$$
\dot{\omega}_{k}=W_{k} \sum_{i=1}^{N_{\text {reac }}} v_{k i} q_{i} \Rightarrow \frac{\partial \dot{\omega}_{k}}{\partial T}=W_{k} \sum_{i=1}^{N_{\text {reac }}} v_{k i} \frac{\partial q_{i}}{\partial T}, \quad \frac{\partial \dot{\omega}_{k}}{\partial \mathfrak{X}_{l}}=W_{k} \sum_{i=1}^{N_{\text {reac }}} v_{k i} \frac{\partial q_{i}}{\partial \mathfrak{X}_{l}}
$$

- Derivatives of rate-of-progress variables

$$
q_{i}=\mathcal{C}_{i} \mathcal{R}_{i} \Rightarrow \frac{\partial q_{i}}{\partial T}=\frac{\partial \mathcal{C}_{i}}{\partial T} \mathcal{R}_{i}+C_{i} \frac{\partial \mathcal{R}_{i}}{\partial T}, \quad \frac{\partial q_{i}}{\partial \mathfrak{X}_{l}}=\frac{\partial \mathcal{C}_{i}}{\partial \mathfrak{X}_{l}} \mathcal{R}_{i}+\mathcal{C}_{i} \frac{\partial \mathcal{R}_{i}}{\partial \mathfrak{X}_{l}}
$$

- Derivatives of $C_{i}$

1. Basic reactions $C_{i}=1: \frac{\partial c_{i}}{\partial T} \equiv \frac{\partial \mathcal{C}_{i}}{\partial \mathfrak{X}_{l}} \equiv 0$

2. $3^{\text {rd }}$ body-enhanced reactions $C_{i}=\mathfrak{X}_{i}: \frac{\partial c_{i}}{\partial T} \equiv 0, \frac{\partial c_{i}}{\partial \mathfrak{X}_{l}}=\alpha_{i l}$

3. Unimolecular/recombination fall-off reactions $C_{i}=\frac{P_{r i}}{1+P_{r i}} F_{i}$

$$
\begin{aligned}
\frac{\partial C_{i}}{\partial T} & =\frac{1}{\left(1+P_{r i}\right)^{2}} \frac{\partial P_{r i}}{\partial T} F_{i}+\frac{P_{r i}}{1+P_{r i}} \frac{\partial F_{i}}{\partial T} \\
\frac{\partial C_{i}}{\partial \mathfrak{X}_{l}} & =\frac{1}{\left(1+P_{r i}\right)^{2}} \frac{\partial P_{r i}}{\partial \mathfrak{X}_{l}} F_{i}+\frac{P_{r i}}{1+P_{r i}} \frac{\partial F_{i}}{\partial \mathfrak{X}_{l}}
\end{aligned}
$$

(a) $P_{r}=\frac{k_{0 i}}{k_{\infty i}} \mathfrak{X}_{i} \Rightarrow \frac{\partial P_{r i}}{\partial T}=\frac{k_{0 i}^{\prime} k_{\infty i}-k_{0 i} k_{\infty i}^{\prime}}{k_{\infty i}^{2}} \mathfrak{X}_{i}, \quad \frac{\partial P_{r i}}{\partial \mathfrak{X}_{l}}=\frac{k_{0 i}}{k_{\infty i}} \alpha_{i l}$.

(b) $P_{r i}=\frac{k_{0 i}}{k_{\infty i}} \mathfrak{X}_{m} \Rightarrow \frac{\partial P_{r i}}{\partial T}=\frac{k_{0 i}^{\prime} k_{\infty i}-k_{0 i} k_{\infty i}^{\prime}}{k_{\infty i}^{2}} \mathfrak{X}_{m}, \quad \frac{\partial P_{r i}}{\partial \mathfrak{X}_{l}}=\frac{k_{0 i}}{k_{\infty i}} \delta_{l m}$, where $\boldsymbol{\delta}_{l m}$ is Kroenecker delta symbol.

(c) For Lindemann form $F_{i}=1 \Rightarrow \frac{\partial F_{i}}{\partial T} \equiv \frac{\partial F_{i}}{\partial \mathfrak{X}_{l}} \equiv 0$.

(d) For Troe form

$$
\begin{aligned}
\frac{\partial F}{\partial T} & =\frac{\partial F}{\partial F_{c e n t}} \frac{\partial F_{c e n t}}{\partial T}+\frac{\partial F}{\partial P_{r}} \frac{\partial P_{r}}{\partial T} \\
\frac{\partial F}{\partial \mathfrak{X}_{l}} & =\frac{\partial F}{\partial F_{c e n t}} \frac{\partial F_{c e n t}}{\partial \mathfrak{X}_{l}}+\frac{\partial F}{\partial P_{r}} \frac{\partial P_{r}}{\partial \mathfrak{X}_{l}}=\frac{\partial F}{\partial P_{r}} \frac{\partial P_{r}}{\partial \mathfrak{X}_{l}} \\
\frac{\partial F}{\partial F_{c e n t}} & =\frac{F}{F_{\text {cent }}\left(1+\left(\frac{A}{B}\right)^{2}\right)}-F \ln F_{\text {cent }}\left(\frac{2 A}{B^{3}}\right) \frac{A_{F} B-B_{F} A}{\left(1+\left(\frac{A}{B}\right)^{2}\right)^{2}} \\
\frac{\partial F}{\partial P_{r}} & =F \ln F_{\text {cent }}\left(\frac{2 A}{B^{3}}\right) \frac{A_{P_{r}} B-B_{P_{r}} A}{\left(1+\left(\frac{A}{B}\right)^{2}\right)^{2}}
\end{aligned}
$$

where

$$
\begin{aligned}
A_{F} & =\frac{\partial A}{\partial F_{\text {cent }}}=-\frac{0.67}{F_{\text {cent }} \ln 10}, \quad B_{F}=\frac{\partial B}{\partial F_{\text {cent }}}=-\frac{1.1762}{F_{\text {cent }} \ln 10} \\
A_{P_{r}} & =\frac{\partial A}{\partial P_{r}}=\frac{1}{P_{r} \ln 10}, \quad B_{P_{r}}=\frac{\partial B}{\partial P_{r}}=-\frac{0.14}{P_{r} \ln 10} \\
\frac{\partial F_{c e n t}}{\partial T} & =-\frac{1-a}{T^{* * *}} \exp \left(-\frac{T}{T^{* * *}}\right)-\frac{a}{T^{*}} \exp \left(-\frac{T}{T^{*}}\right)+\frac{T^{* *}}{T^{2}} \exp \left(-\frac{T^{* *}}{T}\right)
\end{aligned}
$$


(e) For SRI form

$$
\begin{aligned}
\frac{\partial F}{\partial T}= & F\left(\frac{e}{T}+\frac{\partial X}{\partial P_{r}} \frac{\partial P_{r}}{\partial T} \ln \left(a \exp \left(-\frac{b}{T}\right)+\exp \left(-\frac{T}{c}\right)\right)\right. \\
& \left.+X \frac{\frac{a b}{T^{2}} \exp \left(-\frac{b}{T}\right)-\frac{1}{c} \exp \left(-\frac{T}{c}\right)}{a \exp \left(-\frac{b}{T}\right)+\exp \left(-\frac{T}{c}\right)}\right) \\
\frac{\partial F}{\partial \mathfrak{X}_{l}}= & F \ln \left(a \exp \left(-\frac{b}{T}\right)+\exp \left(-\frac{T}{c}\right)\right) \frac{\partial X}{\partial P_{r}} \frac{\partial P_{r}}{\partial \mathfrak{X}_{l}} \\
\frac{\partial X}{\partial P_{r}}= & -X^{2} \frac{2 \log _{1} 0 P_{r}}{P_{r} \ln 10}
\end{aligned}
$$

4. Chemically activated bimolecular reactions: $\mathcal{C}_{i}=\frac{1}{1+P_{r i}} F_{i}$

$$
\begin{aligned}
\frac{\partial C_{i}}{\partial T} & =-\frac{1}{\left(1+P_{r i}\right)^{2}} \frac{\partial P_{r i}}{\partial T} F_{i}+\frac{1}{1+P_{r i}} \frac{\partial F_{i}}{\partial T} \\
\frac{\partial C_{i}}{\partial \mathfrak{X}_{l}} & =-\frac{1}{\left(1+P_{r i}\right)^{2}} \frac{\partial P_{r i}}{\partial \mathfrak{X}_{l}} F_{i}+\frac{1}{1+P_{r i}} \frac{\partial F_{i}}{\partial \mathfrak{X}_{l}}
\end{aligned}
$$

Partial derivatives of $P_{r i}$ and $F_{i}$ are computed similar to the ones above.

- Derivatives of $R_{i}$

$$
\begin{aligned}
\frac{\partial \mathcal{R}_{i}}{\partial T} & =k_{f_{i}}^{\prime} \prod_{j=1}^{N_{\text {spec }}} \mathfrak{X}_{j}^{v_{j i}^{\prime}}-k_{r i}^{\prime} \prod_{j=1}^{N_{\text {spec }}} \mathfrak{X}_{j}^{v_{j i}^{\prime \prime}} \\
\frac{\partial \mathcal{R}_{i}}{\partial \mathfrak{X}_{l}} & =\frac{k_{f_{i}}{v_{l i}^{\prime}}_{l i}^{N_{\text {spec }}} \mathfrak{X}_{j}^{v_{j i}^{\prime}}}{\mathfrak{X}_{l}}-\frac{k_{r i} v_{l i}^{\prime \prime} \prod_{j=1}^{N_{\text {spec }}} \mathfrak{X}_{j}^{v_{j i}^{\prime \prime}}}{\mathfrak{X}_{l}}
\end{aligned}
$$

1. $k_{f_{i}}=A_{i} T^{\beta_{i}} \exp \left(-\frac{E_{a i}}{\Re T}\right)=A_{i} \exp \left(\beta_{i} \ln T-\frac{T_{a i}}{T}\right)$, where $T_{a i}=E_{a i} / \Re$. The derivative with respect to temperature can be calculated as $k_{f_{i}}^{\prime}=\frac{k_{f_{i}}}{T}\left(\beta_{i}+\frac{T_{a i}}{T}\right)$

2. If reverse Arrhenius parameters are provided, $k_{r i}^{\prime}$ is computed similar to above. If $k_{r i}$ is computed based on $k_{f_{i}}$ and the equilibrium constant $K_{c i}$, then its derivative is

$$
\begin{aligned}
k_{r i}=\frac{k_{f_{i}}}{K_{c i}} \Rightarrow k_{r i}^{\prime} & =\frac{k_{f_{i}}^{\prime} K_{c i}-k_{f_{i}} K_{c_{i}}^{\prime}}{K_{c_{i}}^{2}}=\frac{\frac{k_{f_{i}}}{T}\left(\beta_{i}+\frac{T_{a i}}{T}\right)}{K_{c i}}-\frac{k_{f_{i}}}{K_{c i}} \frac{K_{c i}^{\prime}}{K_{c i}} \\
& =k_{r i}\left(\frac{1}{T}\left(\beta_{i}+\frac{T_{a i}}{T}\right)-\frac{K_{c i}^{\prime}}{K_{c i}}\right) .
\end{aligned}
$$

Since $K_{c i}=\left(\frac{p_{a t m}}{\Re}\right)^{\sum_{k=1}^{N_{\text {spec }}} v_{k i}} \exp \left(\sum_{k=1}^{N_{\text {spec }}} v_{k i} g_{k}\right) \Rightarrow \frac{K_{c i}^{\prime}}{K_{c i}}=\sum_{k=1}^{N_{\text {spec }}} v_{k i} g_{k}^{\prime}$. It follows that

$$
k_{r i}^{\prime}=k_{r i}\left(\frac{1}{T}\left(\beta_{i}+\frac{T_{a i}}{T}\right)-\sum_{k=1}^{N_{\mathrm{spec}}} \mathrm{v}_{k i} g_{k}^{\prime}\right)
$$


where $g_{k}^{\prime}$ is computed based on NASA polynomial fits as

$$
g_{k}^{\prime}=\frac{1}{T}\left(a_{0, k}-1+\frac{a_{5, k}}{T}\right)+\frac{a_{1, k}}{2}+T\left(\frac{a_{2, k}}{3}+T\left(\frac{a_{3, k}}{4}+\frac{a_{4, k}}{5} T\right)\right)
$$

\section{Efficient evaluation of the $\mathcal{F}$ terms}

- Step 1:

$$
\begin{aligned}
\mathcal{F}_{3+i, 2} \equiv & 0 \\
\mathcal{F}_{3+i, 3}= & \frac{1}{\rho} \frac{\partial \dot{\omega}_{i}}{\partial T}=\frac{W_{i}}{\rho}\left[\sum_{j=1}^{N_{\text {reac }}} v_{i j} \frac{\partial C_{j}}{\partial T}\left(\mathcal{R}_{f j}-\mathcal{R}_{j}\right)+\sum_{j=1}^{N_{\text {reac }}} v_{i j} \mathcal{C}_{j}\left(\mathcal{R}_{f_{j}} \frac{k_{f_{j}}^{\prime}}{k_{f_{j}}}-\mathcal{R}_{r_{j}} \frac{k_{r j}^{\prime}}{k_{r j}}\right)\right] \\
\mathcal{F}_{3+i, 3+k}= & \frac{1}{\rho} \frac{\partial \dot{\omega}_{i}}{\partial Y_{k}}=\frac{1}{W_{k}} \frac{\partial \dot{\omega}_{i}}{\partial \mathfrak{X}_{k}}=\frac{W_{i}}{W_{k}}\left[\sum_{j=1}^{N_{\text {reac }}} v_{i j} \frac{\partial \mathcal{C}_{j}}{\partial \mathfrak{X}_{k}}\left(\mathcal{R}_{f_{j}}-\mathcal{R}_{r_{j}}\right)+\sum_{j=1}^{N_{\text {reac }}} v_{i j} \mathcal{C}_{j} \frac{\mathcal{R}_{f_{j}} v_{k j}^{\prime}-\mathcal{R}_{j} v_{k j}^{\prime \prime}}{\mathfrak{X}_{k}}\right], \\
& k=1,2, \ldots, N_{\text {spec }}
\end{aligned}
$$

Here $\mathcal{R}_{f_{j}}$ and $\mathcal{R}_{r_{j}}$ are the forward and reverse parts, respectively of $\mathcal{R}_{j}: \mathcal{R}_{f_{j}}=k_{f_{i}} \prod_{j=1}^{N_{\mathrm{spec}}} \mathfrak{X}_{j}^{v_{j i}^{\prime}}, \mathcal{R}_{r_{j}}=$ $k_{r i} \prod_{j=1}^{N_{\text {spec }}} \mathfrak{X}_{j}^{v_{j i}^{\prime \prime}}$.

- Step 2: Once $\mathcal{F}_{3+i, 3+k}$ are evaluated for all $k$, then $\mathcal{F}_{3+i, 1}$ is computed as

$$
\mathcal{F}_{3+i, 1}=\frac{1}{\rho}\left(\frac{\partial \dot{\omega}_{i}}{\partial \rho}-\frac{\dot{\omega}_{i}}{\rho}\right)=\frac{1}{\rho}\left(-\frac{\dot{\omega}_{k}}{\rho}+\sum_{k=1}^{N_{\text {spec }}} \frac{Y_{k}}{W_{k}} \frac{\partial \dot{\omega}_{i}}{\partial \mathfrak{X}_{k}}\right)=\frac{1}{\rho}\left(-\frac{\dot{\omega}_{k}}{\rho}+\sum_{k=1}^{N_{\text {spec }}} Y_{k} \mathcal{F}_{3+i, 3+k}\right)
$$

- Step 3:

$$
\begin{aligned}
\mathcal{F}_{3,1} & =\frac{1}{\rho c_{p}} \sum_{i=1}^{N_{\text {spec }}} h_{i}\left(\frac{\dot{\omega}_{i}}{\rho}-\frac{\partial \dot{\omega}_{i}}{\partial \rho}\right)=-\frac{1}{c_{p}} \sum h_{i} \mathcal{F}_{3+i, 1} \\
\mathcal{F}_{3,2} & \equiv 0 \\
\mathcal{F}_{3,3} & =\frac{1}{\rho c_{p}}\left[\frac{1}{c_{p}} \frac{\partial c_{p}}{\partial T} \sum_{i=1}^{N_{\text {spec }}} h_{i} \dot{\omega}_{i}-\sum_{i=1}^{N_{\text {spec }}} c_{p_{i}} \dot{\omega}_{i}\right]-\frac{1}{\rho c_{p}} \sum_{i=1}^{N_{\text {spec }}} h_{i} \frac{\partial \dot{\omega}_{i}}{\partial T} \\
& =\frac{1}{\rho c_{p}}\left[\frac{1}{c_{p}} \frac{\partial c_{p}}{\partial T} \sum_{i=1}^{N_{\text {spec }}} h_{i} \dot{\omega}_{i}-\sum_{i=1}^{N_{\text {spec }}} c_{p_{i}} \dot{\omega}_{i}\right]-\frac{1}{c_{p}} \sum_{i=1}^{N_{\text {spec }}} h_{i} \mathcal{F}_{3+i, 3}
\end{aligned}
$$

\section{Analytical expressions for $\bar{g}$}

- Line \#1: Pressure equation. The source term for the pressure equation is assumed constant: $f_{2}=$ $\bar{f}_{2}=$ const.

$$
\overline{\mathcal{g}}_{1, j} \equiv 0 \text { for all } j
$$


- Line \#2: Temperature equation :

$$
\begin{aligned}
\bar{g}_{2,1} & =\mathcal{F}_{3,2}+\mathcal{F}_{3,1} \frac{\partial \rho}{\partial P} \\
\bar{g}_{2,2} & =\mathcal{F}_{3,3}+\mathcal{F}_{3,1} \frac{\partial \rho}{\partial T} \\
\bar{g}_{2,2+k} & =\mathcal{F}_{3,3+k}+\mathcal{F}_{3,1} \frac{\partial \rho}{\partial Y_{k}}
\end{aligned}
$$

- Line $\# \mathrm{i}=3, \ldots, N_{\mathrm{spec}}+2$ : Species equations :

$$
\begin{aligned}
\overline{\mathfrak{g}}_{i, 1} & =\mathcal{F}_{i+1,2}+\mathcal{F}_{i+1,1} \frac{\partial \rho}{\partial P} \\
\bar{g}_{i, 2} & =\mathcal{F}_{i+1,3}+\mathcal{F}_{i+1,1} \frac{\partial \rho}{\partial T} \\
\bar{g}_{i, 2+k} & =\mathcal{F}_{i+1,3+k}+\mathcal{F}_{i+1,1} \frac{\partial \rho}{\partial Y_{k}}, k=1,2, \ldots, N_{\text {spec }}
\end{aligned}
$$

For this case density is a dependent variable, calculated based on the ideal gas equation of state:

$$
\rho=\frac{P}{\Re T \sum_{k=1}^{N_{\text {spec }} Y_{k}} \frac{W_{k}}{W_{k}}}
$$

The partial derivaties of density with respect to the independent variables are computed as

$$
\frac{\partial \rho}{\partial P}=\frac{\rho}{P}, \quad \frac{\partial \rho}{\partial T}=-\frac{\rho}{T}, \quad \frac{\partial \rho}{\partial Y_{k}}=-\frac{\rho \bar{W}}{W_{k}} .
$$

\section{Analytical expressions for $\tilde{g}$}

- Line \#1: Pressure equation. The source term for the pressure equation is assumed constant: $f_{2}=$ $\widetilde{f}_{2}=$ const .

$$
\widetilde{g}_{1, j} \equiv 0 \text { for all } j
$$

- Line \#2: Temperature equation:

$$
\begin{gathered}
\tilde{g}_{2,1}=\mathcal{F}_{3,2}+\mathcal{F}_{3,1} \frac{\partial \rho}{\partial P}+\mathcal{F}_{3, L+3} \frac{\partial Y_{L}}{\partial P} \\
\tilde{g}_{2,2}=\mathcal{F}_{3,3}+\mathcal{F}_{3,1} \frac{\partial \rho}{\partial T}+\mathcal{F}_{3, L+3} \frac{\partial Y_{L}}{\partial T} \\
\tilde{g}_{2,2+k}=\mathcal{F}_{3,3+k_{s}}+\mathcal{F}_{3,1} \frac{\partial \rho}{\partial Y_{k}}+\mathcal{F}_{3, L+3} \frac{\partial Y_{L}}{\partial Y_{k}}, \\
k=1, \ldots, L-1, L+1, \ldots, N_{\text {spec }}
\end{gathered}
$$

where

$$
k_{s}= \begin{cases}k & k<3+L \\ k+1 & \text { otherwise }\end{cases}
$$


- Line $\# \mathrm{i}=3, \ldots, N_{\mathrm{spec}}+1$ : Species equations :

$$
\begin{gathered}
\widetilde{\mathcal{g}}_{i, 1}=\mathcal{F}_{i+i_{s}, 2}+\mathcal{F}_{i+i_{s}, 1} \frac{\partial \rho}{\partial P}+\mathcal{F}_{i+i_{s}, L+3} \frac{\partial Y_{L}}{\partial P} \\
\widetilde{\mathcal{J}}_{i, 2}=\mathcal{F}_{i+i_{s}, 3}+\mathcal{F}_{i+i_{s}, 1} \frac{\partial \rho}{\partial T}+\mathcal{F}_{i+i_{s}, L+3} \frac{\partial Y_{L}}{\partial T} \\
\widetilde{\mathcal{J}}_{i, 2+k}=\mathcal{F}_{i+i_{s}, 3+k_{s}}+\mathcal{F}_{i+i_{s}, 1} \frac{\partial \rho}{\partial Y_{k}}+\mathcal{F}_{i+i_{s}, L+3} \frac{\partial Y_{L}}{\partial Y_{k}}, \\
k=1, \ldots, L-1, L+1, \ldots, N_{\mathrm{spec}}
\end{gathered}
$$

where $k_{s}$ is defined above and

$$
i_{s}= \begin{cases}1 & i+1<3+L \\ 2 & \text { otherwise }\end{cases}
$$

For this case, density and mass fraction of species $L$ are dependent variables, calculated as

$$
\begin{aligned}
\rho & =\frac{P}{\Re T\left(\frac{1}{W_{L}}+\sum_{\substack{k=1 \\
k \neq L}}^{N_{\text {spec }}} Y_{k}\left(\frac{1}{W_{k}}-\frac{1}{W_{L}}\right)\right)} \\
Y_{L} & =1-\sum_{\substack{k=1 \\
k \neq L}}^{N_{\text {spec }}} Y_{k}
\end{aligned}
$$

The partial derivaties of density and $Y_{L}$ with respect to the independent variables are computed as

$$
\begin{aligned}
& \frac{\partial \rho}{\partial P}=\frac{\rho}{P}, \quad \frac{\partial \rho}{\partial T}=-\frac{\rho}{T}, \quad \frac{\partial \rho}{\partial Y_{k}}=-\rho \bar{W}\left(\frac{1}{W_{k}}-\frac{1}{W_{L}}\right) \\
& \frac{\partial Y_{L}}{\partial P}=\frac{\partial Y_{L}}{\partial T}=0, \quad \frac{\partial Y_{L}}{\partial Y_{k}}=-1 .
\end{aligned}
$$




\section{Chapter 5}

\section{Examples}

This section presents example $\mathrm{C}, \mathrm{C}++$, and Fortran 77 example codes that use some of the functionalities provided by TChem. The $\mathrm{C}$ and $\mathrm{C}++$ examples requires the $\mathrm{CVODE}^{1}$ library [2]. The Fortran example requires DVODE ${ }^{2}$ library [1]. All these examples numerically integrate a OD ignition model (1.1) for a range of parameters that control the initial fuel-oxidizer mixture, temperature, and pressure.

\section{ign-c}

The main function of the $\mathrm{C}$ code is contained in ign.c. The main function defines the parameters, then proceeds to set up the simulation: parameter setup (in setup.c), TChem initialization, CVODE setup initcvode.c. The time advancement of the sytem of ODE's (1.1) is implemented in doIgn.c. The function contained in doIgnReinit.c is a more compact version of doIgn.c (limited output) and takes advantage of the re-initialization option of CVODE to perform a series of time integrations while the kinetic model parameters are modified. An output function is provided in output.c. Functions providing the rhs and the Jacobian matrix corresponding to (1.1) are implemented in rhsjac.c.

Select sections of the the various components of the $\mathrm{C}$ code are described below.

$\underline{\text { ign.c }}$

- Function TC_initChem is used to initalize TChem, while function TC_setThermoPres sends the thermodynamic pressure to the library.

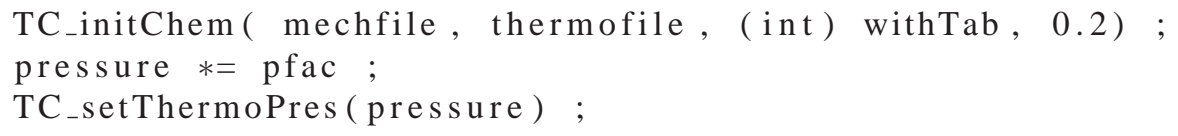

The parameters used in these functions are described below in setup.c.

- Function TC_getSpos returns the position in of a species in the list of species read by TChem from the kinetic model file.

for $(\mathrm{i}=0 ; \mathrm{i}<\operatorname{specinno} ; \mathrm{i}++)$

\{

\footnotetext{
${ }^{1}$ https://computation.llnl.gov/casc/sundials

${ }^{2}$ https://computation.llnl.gov/casc/software.html
} 


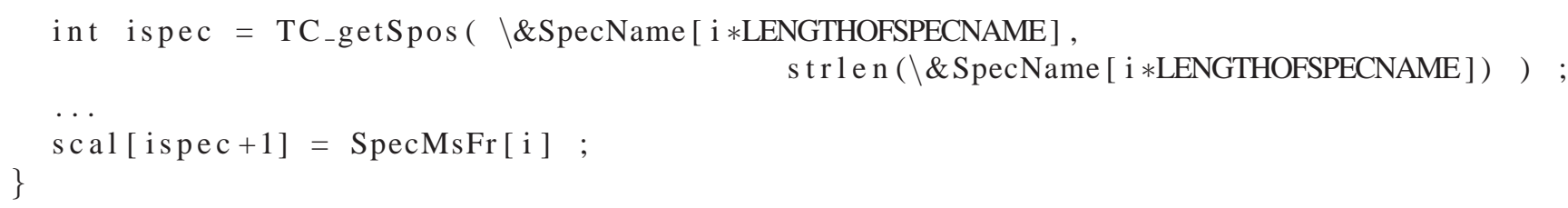

- The ignition code expects mole fractions for the species that enter the fresh mixture. It the converts these values to mass fractions using $T C_{-}$get $M l 2 M s$

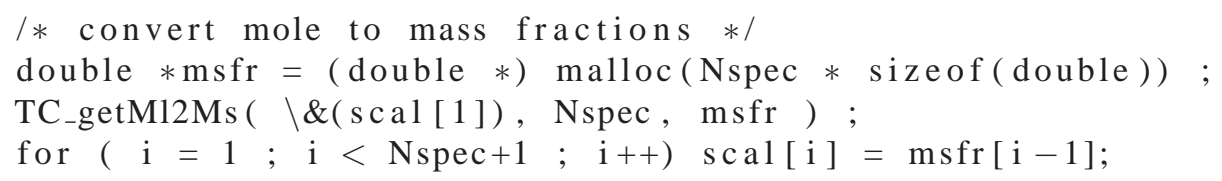

- Before the end of the execution the main function call TC_reset to clear all internal TChem arrays.

\section{setup.c}

The function contained in this file, setup, initializes parameters with default values, the reads from a setup file custom values for the paramters the user wants to change.

Below, NiterMax is the maximum number of time steps, oFreq is the frequency of solution output to disk, Tini is the initial mixture temperature, Temp_id is the temperature threshold for the ignition delay time, deltat is the initial time step size in seconds, deltatMax is the maximum time step size in seconds, $t$ End is the end time for the time advancement, deltaTemp is the maximum allowed temperature change per time step. The thermodynamic pressure is given by the product $\mathrm{pfac} \times 1.01325 \times 10^{5} \mathrm{~Pa}$. CVrelt and CVsmall are tolerances for the CVODE library. The kinetic model file name is stored in mechfile and the name of the file with the NASA polynomials for the thermodynamic properties is stored in thermofile. withTab is a flag that specifies whether TChem should use interpolation tables to compute various properties, while getIgnDel is a flag allows the user to stop the integration once the ignition take place.

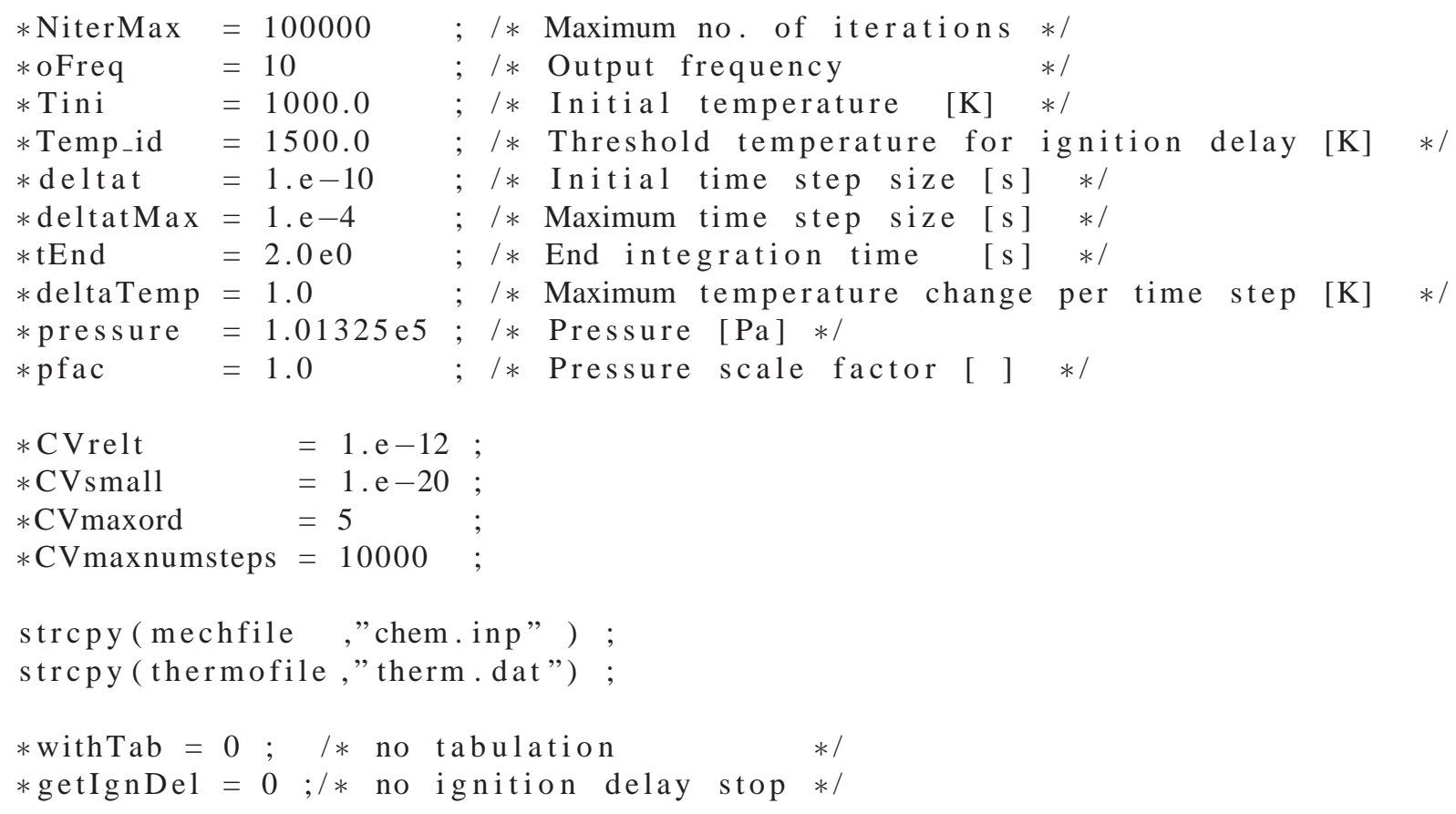



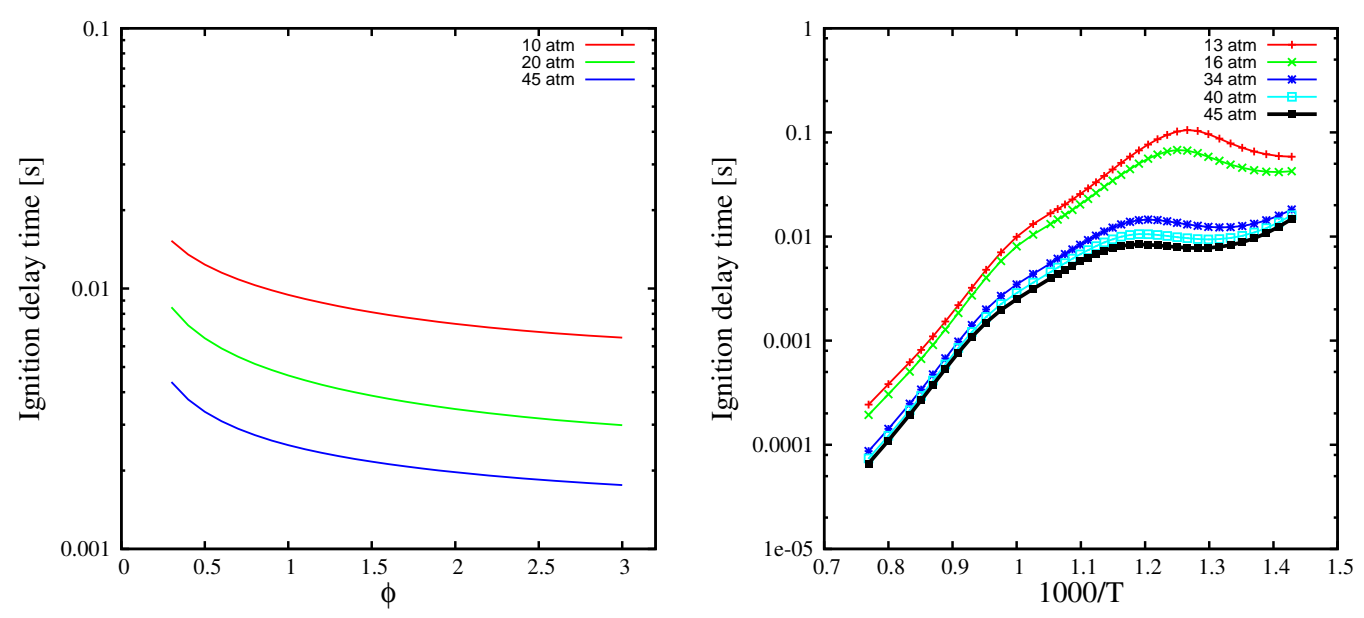

Figure 5.1. Left Frame: variation of the ignition delay times with the equivalence ratio $(\Phi)$ for iso-octane/air mixtures at various pressures and initial temperature of 1000K. Right Frame: Variation of ignition delay time with initial temperature for a stoichiometric iso-octane/mixtures at various pressures. The kinetic model involves 874 species and 3796 reactions [5]

\section{rhsjac.c}

The functions that compute rhs source terms and Jacobian matrices can be used for both full systems (temperature and all species) or restricted systems (temperature + all species - one species). In the later case, the mass fraction of the negelected species is set to ensure mass conservation. The user should specify at compile time "-DALLSPEC" for the former case. If this flag is neglected, the code will compiled for restricted systems.

The algorithms are doIgn.c, doIgnReinit.c, and output.c are straigthforward.

\section{Sample results}

Sample simulations are provided in example/ign-c/run. These simulations can be replicated using the bash scripts located in the corresponding directories. All script files have the extension .x. These scripts can also be used to generate the ignition delay time results for iso-Octane and Primary Reference Fuel (PRF) mixtures shown in Figs. 5.1 and 5.2, respectively. 

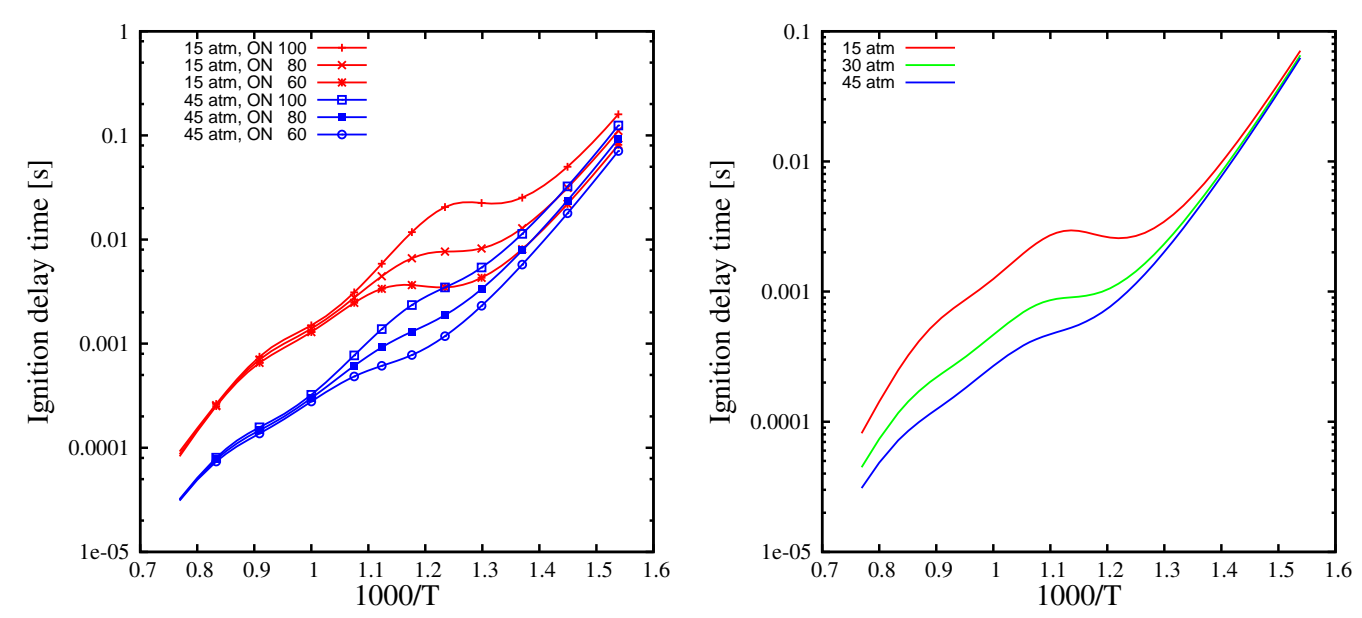

Figure 5.2. Left Frame: Variation of the ignition delay times with the initial mixture temperature for $100 \mathrm{ON}, 80 \mathrm{ON}$ and $60 \mathrm{ON}$ at 15 atm and $45 \mathrm{~atm}$. Right Frame: Variation of ignition delay time for $50 \mathrm{ON}$ at $15 \mathrm{~atm}, 30 \mathrm{~atm}$, and $45 \mathrm{~atm}$. The kinetic model involves 1034 species and 4236 reactions [4, 3]

\section{ign-cpp}

This $\mathrm{C}++$ example code is located in example/ign-cpp/src. Sample simulation data and scripts using a methane kinetic model (53 species, 325 reactions) [7] and a iso-octane kinetic model (871 species, 3792 reactions) [5] are located in example/ign-c/run. Subsequent directories' names are self-explanatory.

The main function of the $\mathrm{C}++$ code is contained in ign.cpp. The main function handles the setup of the simulation, initialization of TChem, then transfers the control to the StiffInteg class. The StiffInteg class implements functions for the right hand side ( $r h s)$ and Jacobian matrix corresponding to the system of ordinarry differential equations 1.1. It handles the setup of CVODE and controls the time integration of 1.1. Select code sections are described below:

$\underline{\text { ign.cpp }}$

- Setup parameters. Below, NiterMax is the maximum number of time steps, oFreq is the frequency of solution output to disk, Tini is the initial mixture temperature, deltat is the initial time step size in seconds, deltatMax is the maximum time step size in seconds, tEnd is the end time for the time advancement, deltaTemp is the maximum allowed temperature change per time step. The thermodynamic pressure is given by the product $\mathrm{pfac} \times 1.01325 \times 10^{5} \mathrm{~Pa}$. CVrelt and CVsmall are tolerances for the CVODE library. The kinetic model file name is stored in mechfile and the name of the file with the NASA polynomials for the thermodynamic properties is stored in thermofile. withTab is a flag that specifies whether TChem should use interpolation tables to compute various properties.

I* Set default values $* /$ 


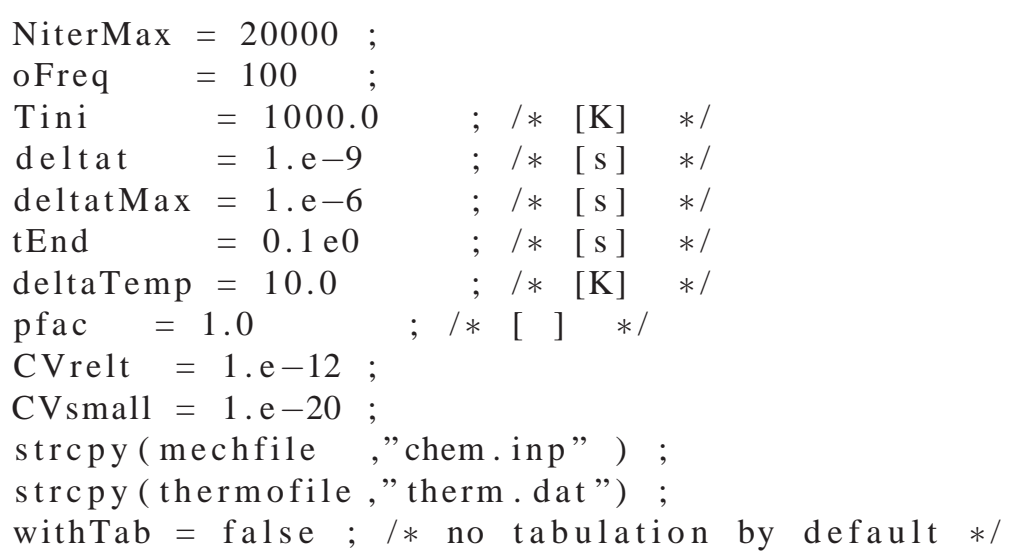

The default values of these parameters can be changed through the setup file. In addition to these parameters the setup files should contain mole fractions for the initial fuel-oxidizer mixture. Each species mole fractions should be provided on separate lines, starting with the keyword spec followed by the species name, and the mole fraction value. Examples setup files, "input.setup" are provided for each example.

- Function TC_initChem is used to initalize TChem, while function TC_setThermoPres sends the thermodynamic pressure to the library.

TC_initChem( mechfile, thermofile, (int) withTab, 0.2) ;

pressure $*=$ pfac ;

TC_setThermoPres(pressure) ;

- The constructor for the StiffInteg class requires a pointer to the initial condition array scal, the number of species Nspec, and the maximum temperature change per time step.

StiffInteg intgcrode( scal, Nspec, deltaTemp ) ;

- The compute function of StiffInteg handles the time advancement of the system of ordinary differential equations (1.1).

intgcvode.compute( tEnd, \&deltat, deltatMax, NiterMax, oFreq ) ;

\section{StiffInteg.cpp}

- Function StiffInteg::chemrhs handles the computation of the rhs source terms through a call to TChem. StiffInteg::chemrhs can handle chemical systems that include all species, $\left(T, Y_{1}, \ldots, Y_{N_{\text {spec }}}\right)$, or alternatively $\left(T, Y_{1}, \ldots, Y_{N_{\text {spec }}-1}\right)$. In the later case, the mass fraction of the last species is computed as $Y_{N_{\text {spec }}}=1-\sum_{k=1}^{N_{\text {spec }}-1} Y_{k}$. Below, tempNmsfr is the pointer to the array holding the temperature and species mass fractions, and rhsvals is the pointer to the array that stores the rhs values.

TC_getSrc ( tempNmsfr, Nspec_+1, rhsvals ) ;

- Function StiffInteg::chemjac handles the computation of the Jacobian matrix . Similar to StiffInteg::chemrhs, this function can provide matrices for both types of chemical systems mentioned above, by appropriate calls to the TChem library. Below, tempNmsfr is the pointer to the array holding the temperature and species mass fractions, jactmp is the pointer to the array that stores the Jacobian matrix, and useJacAnl is a flag which indicates whether or not to use analytical expressions for the Jacobian matrix terms (default is analytical). 

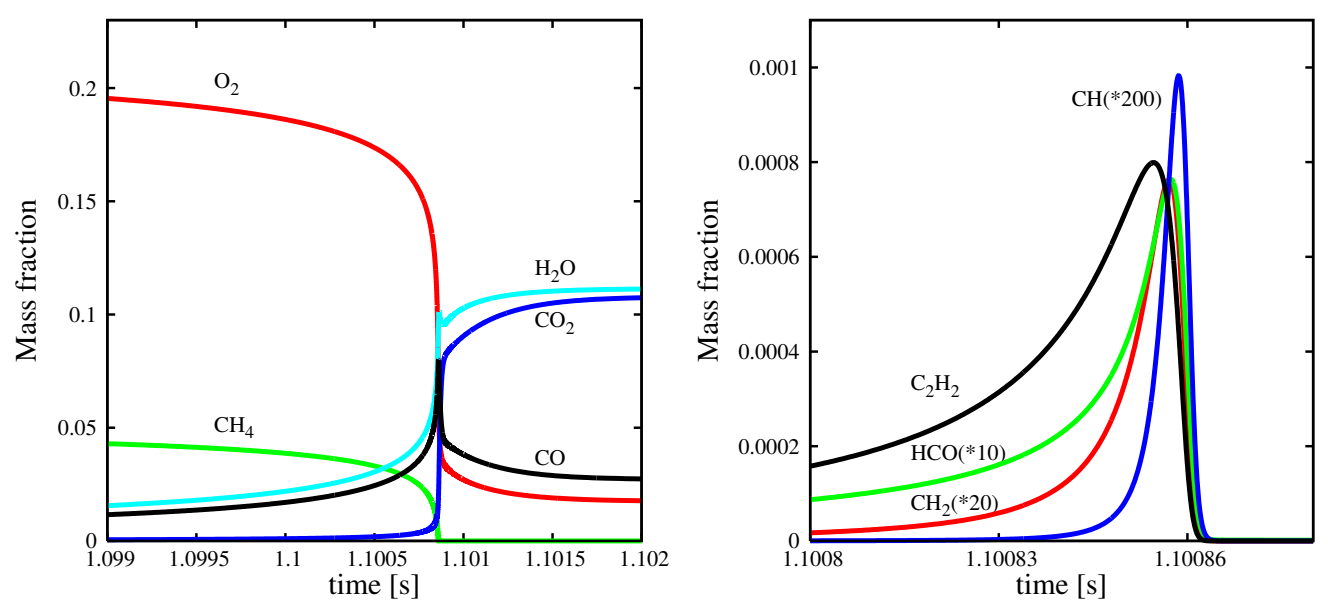

Figure 5.3. Mass fraction profiles during the constant pressure ignition of a stoichimetric methane-air mixture at $1 \mathrm{~atm}$. Methane combustion is modeled using the GRI-Mech v3.0 kinetic model (53 species, 325 reactions) [7].

TC_getJacTYN ( tempNmsfr, Nspec _, jactmp, (unsigned int) useJacAnl );

TC_getJacTYNm1 ( tempNmsfr, Nspec -, jactmp, (unsigned int) useJacAnl ) ;

- StiffInteg::compute outputs data to several files during the time advancement: (1) ignsol.dat contains on each row the time $[\mathrm{s}]$, time step $[\mathrm{s}]$, temperature $[\mathrm{K}]$, and species mass fractions, (2) ys.out contains the time [s], temperature [K], and species mass fractions, (3) cs.out contains the time [s], temperature $[\mathrm{K}]$, and species molar concentrations $\left[\mathrm{kmol} / \mathrm{m}^{3}\right]$, and (4) h.out contains the time $[\mathrm{s}]$ and mixture specific enthalpy $[\mathrm{J} / \mathrm{kg}]$.

\section{Sample results}

Sample simulations are provided in example/ign-cpp/run. These simulations can be replicated using the bash scripts located in the corresponding directories. All script files have the extension .x. These scripts can also be used to generate the graphs shown in Fig. 5.3.

\section{ign-f}

The Fortran 77 example code is located in example/ign-f/src. Sample simulation data and scripts using a methane kinetic model (53 species, 325 reactions) and a one step model are located in example/ign-f/run. Subsequent directories' names are self-explanatory.

Most of the Fortran code is contained in ign.f. The variable names are similar to the ones described 
above for ign-c. The subroutines that connect to TChem for the calculation of the rhs values and the Jacobian matrices are located in rhsjac.f

Note that in the subroutines calls that originate in the Fortran code, all parameters are passed by reference. In the TChem C-library, most functions contain parameters passed by reference as well as passed by value. In order to facilitate the inter-language calls, an additional interface is placed between the Fortran code and the TChem. The functions in this interface have all parameters passed by reference and forward the calls to the corresponding main $\mathrm{C}$-interface with select parameters passed by value.

The sample Fortran code below, extracted from ign.f, is used to illustrate the above methodology. Here, tcgetarhenfor is used to extract the value of the activation energy for reaction \# 0 from TChem. The value is modified, then it is sent back using tcchgarhenfor.

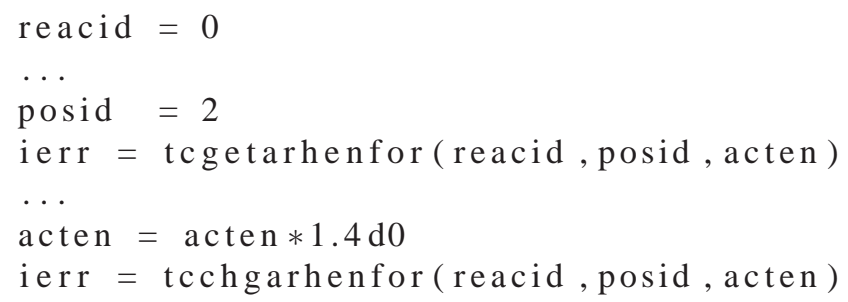

The definition for tcgetarhenfor, extracted from file TC for.c is self explanatory:

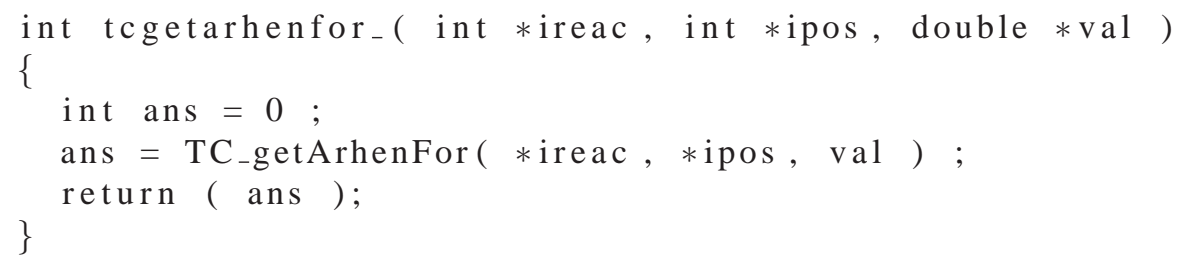

The interface functions that facilitate the calls between Fortran codes and TChem are located in TC for.c. 
This page intentionally left blank. 


\section{Chapter 6}

\section{Library Functions}

\section{TC_chg.c}

1. int TC_chgArhenFor(int $i_{\text {reac }}$, int $i_{\text {pos }}$, double newval)

Change parameters for forward rate constants.

- $i_{\text {reac }}$ - reaction index.

- $i_{\text {pos }}$ - index of parameter to be changed (0) pre-exponential factor (1) temperature exponent, (2) activation energy

- newval - new parameter value

2. int $\mathbf{T C}$ _chgArhenForBack(int $i_{\text {reac }}$, int $\left.i_{\text {pos }}\right)$

Reverse changes for forward rate constants' parameters

- $i_{\text {reac }}$ - reaction index.

- $i_{\text {pos }}$ - index of parameter to be changed (0) pre-exponential factor (1) temperature exponent, (2) activation energy

3. int TC_chgArhenRev(int $i_{\text {reac }}$, int $i_{\text {pos }}$, double newval)

Change parameters for forward rate constants.

- $i_{\text {reac }}$ - reaction index.

- $i_{\text {pos }}$ - index of parameter to be changed (0) pre-exponential factor (1) temperature exponent, (2) activation energy

- newval - new parameter value

4. int $\mathbf{T C}$ _chgArhenRevBack(int $i_{\text {reac }}$, int $\left.i_{\text {pos }}\right)$

Reverse changes for forward rate constants' parameters

- $i_{\text {reac }}$ - reaction index.

- $i_{\text {pos }}$ - index of parameter to be changed (0) pre-exponential factor (1) temperature exponent, (2) activation energy 


\section{1. int $\mathbf{T C} \_$reset()}

Frees all memory and sets variables to 0 so that TC_initChem can be called again without a memory leak. Not designed for use with tables.

\section{TC init.c}

1. int TC_initChem(char *mechfile,char *thermofile, int tab, double delT) Initializes the library:

- mechfile - file containing the kinetic model ( in chemkin format )

- thermofile - file containing the coefficients for NASA polynomials.

- tab - flag: 0-use direct evaluations to compute various properties, 1-use interpolation tables

- delT - temperature step size for the interpolation tables; not used if tab=0

2. void TC_setRefVal(double $r h o_{\text {ref }}$, double $p_{\text {ref }}$, double $T_{\text {ref }}$, double $W_{\text {ref }}$, double $D a_{\text {ref }}$, double $o m g_{\text {ref }}$, double $c p_{\text {ref }}$, double $h_{\text {ref }}$, double $t i m_{\text {ref }}$ )

Send reference values to the library:

- these are the density $\left(r h o_{\text {ref }}\right)$, pressure $\left(p_{\text {ref }}\right)$, temperature $\left(T_{\text {ref }}\right)$, molecular weight $\left(W_{\text {ref }}\right)$, Damkohler number $\left(D a_{\text {ref }}\right)$, reaction rate $\left(o m g_{\text {ref }}\right)$, specific heat at constant pressure $\left(c p_{\text {ref }}\right)$, specific enthalpy $\left(h_{\text {ref }}\right)$, time $\left(\right.$ tim $\left._{\text {ref }}\right)$.

3. void TC_setNonDim()

Set's the library to use non-dimensional input and functions. Can be called only after TC_setRefVal was called.

4. void TC_setDim()

Set's the library to use dimensional input and functions.

5. void TC_setThermoPres(double pressure)

Sends the thermodynamic pressure to the library.

- pressure - dimensiona/non-dimensional thermodynamic pressure. For dimensional cases, SI units are used $\left[\mathrm{N} / \mathrm{m}^{2}\right]$

\section{TC_mlms.c}

1. int TCDND_getMs2Cc(double $*^{*} s c a l$,int $N_{\text {vars }}$, double $*^{*}$ concX)

Computes molar concentrations $\mathfrak{X}$ 's based on temperature $T$ and species mass fractions $Y$ 's.

$$
\mathfrak{X}_{k}=Y_{k} \cdot \frac{\rho}{W_{k}}
$$


If the non-dimensional flag is $\mathrm{ON}$ (using TC_setNonDim) this function expects non-dimensional input and will provide non-dimensional output.

- scal - pointer to an array of $N_{\text {spec }}+1$ doubles $\left(T, Y_{1}, Y_{2}, \ldots, Y_{N}\right)$, temperature $T[\mathrm{~K}]$, mass fractions $Y$ [].

- $N_{\text {vars }}$ - no. of variables $=N_{\text {spec }}+1$

- $\operatorname{concX}$ - array of doubles containing species molar concentrations $\mathfrak{X}$ 's $\left[\mathrm{kmol} / \mathrm{m}^{3}\right]$

2. int TC_getMs2Cc(double *scal, int $N_{\text {vars }}$, double *concX)

Computes molar concentrations $\mathfrak{X}$ 's based on temperature $T$ and species mass fractions $Y$ 's.

$$
\mathfrak{X}_{k}=Y_{k} \cdot \frac{\rho}{W_{k}}
$$

- scal - pointer to an array of $N_{\text {spec }}+1$ doubles $\left(T, Y_{1}, Y_{2}, \ldots, Y_{N}\right)$, temperature $T[\mathrm{~K}]$, mass fractions $Y[]$.

- $N_{\text {vars }}$ - no. of variables $=N_{\text {spec }}+1$

- $\operatorname{concX}$ - array of doubles containing species molar concentrations [ $\left.\mathrm{kmol} / \mathrm{m}^{3}\right]$

3. int TCDND_getMl2Ms(double $* X s p e c$,int $N_{\text {spec }}$, double $* Y$ spec $)$

Transforms mole fractions $X$ 's to mass fractions $Y$ 's (same as TC_getMl2Ms()).

$$
Y_{k}=X_{k} \cdot W_{k} / \bar{W}
$$

- Xspec - array of $N_{\text {spec }}$ mole fractions $X$ [].

- $N_{\text {spec }}$ - no. of species

- $Y$ spec - array of $N_{\text {spec }}$ mole fractions $Y$ [].

4. int TC_getMl2Ms(double *Xspec,int $N_{\text {spec }}$, double *Yspec)

Transforms mole fractions $X$ 's to mass fractions $Y$ 's.

$$
Y_{k}=X_{k} \cdot W_{k} / \bar{W}
$$

- Xspec - array of $N_{\text {spec }}$ mole fractions $X$ [].

- $N_{\text {spec }}$ - no. of species

- Yspec - array of $N_{\text {spec }}$ mole fractions $Y$ [].

5. int TCDND_getMs2Ml(double $* Y$ spec, int $N_{\text {spec }}$, double $\left.* X s p e c\right)$

Transforms mass fractions $Y$ 's to mole fractions $X$ 's (same as TC_getMs $2 \mathrm{Ml}()$ ).

$$
X_{k}=Y_{k} \cdot \bar{W} / W_{k}
$$

- $Y$ spec - array of $N_{\text {spec }}$ mole fractions $Y$ [].

- $N_{\text {spec }}-$ no. of species

- Xspec - array of $N_{\text {spec }}$ mole fractions $X$ []. 
6. int TC_getMs2Ml(double $* Y$ spec, int $N_{\text {spec }}$, double $\left.* X s p e c\right)$

Transforms mass fractions $Y$ 's to mole fractions $X$ 's.

$$
X_{k}=Y_{k} \cdot \bar{W} / W_{k}
$$

- $Y$ spec - array of $N_{\text {spec }}$ mole fractions $Y$ [].

- $N_{\text {spec }}$ - no. of species

- Xspec - array of $N_{\text {spec }}$ mole fractions $X$ [].

7. int TCDND_getMs2Wmix(double $* Y$ spec, int $N_{\text {spec }}$, double $\left.* W m i x\right)$

Computes mixture molecular weight $\bar{W}$ based on species mass fractions $Y$ 's. If the non-dimensional flag is ON (using TC_setNonDim) this function expects non-dimensional input and will provide non-dimensional output.

$$
\bar{W}=\left(\sum_{k=1}^{N_{s p e c}} Y_{k} / W_{k}\right)^{-1}
$$

- $Y$ spec - array of $N_{\text {spec }}$ mole fractions $Y[]$.

- $N_{\text {spec }}$ - no. of species

- Wmix - pointer to mixture molecular weight $[\mathrm{kg} / \mathrm{kmol}]=[\mathrm{g} / \mathrm{mol}]$.

8. int TC_getMs2Wmix(double $* Y$ spec, int $N_{\text {spec }}$, double $\left.* W m i x\right)$

Computes mixture molecular weight $\bar{W}$ based on species mass fractions $Y$ 's.

$$
\bar{W}=\left(\sum_{k=1}^{N_{s p e c}} Y_{k} / W_{k}\right)^{-1}
$$

- $Y$ spec - array of $N_{\text {spec }}$ mole fractions $Y[]$.

- $N_{\text {spec }}$ - no. of species

- Wmix - pointer to mixture molecular weight $[\mathrm{kg} / \mathrm{kmol}]=[\mathrm{g} / \mathrm{mol}]$.

9. int TCDND_getMl2Wmix(double $* X s p e c$, int $N_{\text {spec }}$, double $\left.* W m i x\right)$

Computes mixture molecular weight $\bar{W}$ based on species mole fractions $X$ 's. If the non-dimensional flag is ON (using TC_setNonDim) this function expects non-dimensional input and will provide non-dimensional output.

$$
\bar{W}=\frac{1}{W_{\text {ref }}} \sum_{k=1}^{N_{\text {spec }}} X_{k} W_{k}
$$

- Xspec - array of $N_{\text {spec }}$ mole fractions $X$ [].

- $N_{\text {spec }}$ - no. of species

- Wmix - pointer to mixture molecular weight $[\mathrm{kg} / \mathrm{kmol}]=[\mathrm{g} / \mathrm{mol}]$. 
10. int TC_getMl2Wmix (double $* X$ spec, int $N_{\text {spec }}$, double $\left.* W m i x\right)$

Computes mixture molecular weight $\bar{W}$ based on species mole fractions $X$ 's.

$$
\bar{W}=\sum_{k=1}^{N_{\text {spec }}} X_{k} W_{k}
$$

- Xspec - array of $N_{\text {spec }}$ mole fractions $X$ [].

- $N_{\text {spec }}$ - no. of species

- Wmix - pointer to mixture molecular weight $[\mathrm{kg} / \mathrm{kmol}]=[\mathrm{g} / \mathrm{mol}]$.

\section{TC_rr.c}

1. int $\mathbf{T C}$ _getNreac()

Returns number of reactions $N_{\text {reac }}$.

2. int TC_getStoiCoef(int $N_{\text {spec }}$, int $N_{\text {reac }}$, double *stoicoef)

Returns stoichiometric coefficients' matrix. The stoichiometric coefficient for species $j$ in reaction $i$ is stored at position $i \cdot N_{\text {spec }}+j$. It assumes that stoicoef was dimentioned to at least $N_{\text {reac }} \times N_{\text {spec }}$

- $N_{\text {spec }}$ - no. of species

- $N_{\text {reac }}$ - no. of reactions

- stoicoef - pointer to an array of doubles with the stoichiometric coefficients.

3. int TC_getStoiCoefReac(int $N_{\text {spec }}$, int $N_{\text {reac }}$, int $i_{\text {reac }}$, int idx, double $*_{\text {stoicoef }}$

Returns stoichiometric coefficients' array for reaction $i_{\text {reac }}$ for either reactants $(i d x=0)$ or products $(i d x=1)$. The stoichiometric coefficient for species $j$ in reaction $i_{\text {reac }}$ is stored at position $j$. It assumes that stoicoef was dimentioned to at least $N_{\text {spec }}$

- $N_{\text {spec }}$ - no. of species

- $N_{\text {reac }}-$ no. of reactions

- $i_{\text {reac }}$ - reaction index

- idx - 0-reactants, 1-products

- stoicoef - pointer to an array of doubles with the stoichiometric coefficients.

4. int TC_getArhenFor(int $i_{\text {reac }}$, int $i_{\text {pos }}$, double *val)

Return current value of the Arrhenius parameters for forward rate constants. Return - 1 if no data available, otherwise return 0 and store value in val.

- $i_{\text {reac }}$ - reaction index.

- $i_{\text {pos }}$ - index of Arrhenius parameter (0) pre-exponential factor (1) temperature exponent, (2) activation energy

- val - pointer to the value of Arrhenius parameter. 
5. int $\mathbf{T C}$ _getArhenRev(int $i_{\text {reac }}$, int $i_{\text {pos }}$, double $*$ val $)$

Return current value of Arrhenius parameters for reverse rate constants. Return - 1 if no data available, otherwise return 0 and store value in val.

- $i_{\text {reac }}$ - reaction index.

- $i_{\text {pos }}$ - index of Arrhenius parameter (0) pre-exponential factor (1) temperature exponent, (2) activation energy

- $v a l$ - pointer to the value of Arrhenius parameter

6. int TCDND_getTY2RRml(double *scal, int $N_{\text {vars }}$, double *omega)

Returns molar reaction rates, $\dot{\omega}_{i}$, based on temperature $T$ and mass fractions $Y$ 's. If the nondimensional flag is ON (using TC_setNonDim) this function expects non-dimensional input and will provide non-dimensional output.

- scal - pointer to an array of $N_{\text {spec }}+1$ doubles $\left(T, Y_{1}, Y_{2}, \ldots, Y_{N}\right)$, temperature $T$ [K], mass fractions $Y$ [].

- $N_{\text {vars }}$ - no. of variables $=N_{\text {spec }}+1$

- omega - array of $N_{\text {spec }}$ molar reaction rates $\left[\mathrm{kmol} /\left(\mathrm{m}^{3} \cdot \mathrm{s}\right)\right]$

7. int TC_getTY2RRml(double *scal, int $N_{\text {vars }}$, double *omega)

Returns molar reaction rates, $\dot{\omega}_{i}$, based on $T$ and $Y$ 's.

- scal - pointer to an array of $N_{\text {spec }}+1$ doubles $\left(T, Y_{1}, Y_{2}, \ldots, Y_{N}\right)$, temperature $T$ [K], mass fractions $Y$ [].

- $N_{\text {vars }}$ - no. of variables $=N_{\text {spec }}+1$

- omega - array of $N_{\text {spec }}$ molar reaction rates $\dot{\omega}_{i}\left[\mathrm{kmol} /\left(\mathrm{m}^{3} \cdot \mathrm{s}\right)\right]$.

8. int TCDND_getTY2RRms(double *scal, int $N_{\text {vars }}$, double *omega)

Returns mass reaction rates based on temperature $T$ and species mass fractions $Y$ 's. If the nondimensional flag is ON (using TC_setNonDim) this function expects non-dimensional input and will provide non-dimensional output.

- scal - pointer to an array of $N_{\text {spec }}+1$ doubles $\left(T, Y_{1}, Y_{2}, \ldots, Y_{N}\right)$, temperature $T$ [K], mass fractions $Y$ [].

- $N_{\text {vars }}$ - no. of variables $=N_{\text {spec }}+1$

- omega - array of $N_{\text {spec }}$ mass reaction rates $\left[\mathrm{kg} /\left(\mathrm{m}^{3} \cdot \mathrm{s}\right)\right]$

9. int TC_getTY2RRms(double *scal, int $N_{\text {vars }}$, double *omega)

Returns mass reaction rates based on temperature $T$ and species mass fractions $Y$ 's.

- scal - pointer to an array of $N_{\text {spec }}+1$ doubles $\left(T, Y_{1}, Y_{2}, \ldots, Y_{N}\right)$, temperature $T[\mathrm{~K}]$, mass fractions $Y$ [].

- $N_{\text {vars }}$ - no. of variables $=N_{\text {spec }}+1$

- omega - array of $N_{\text {spec }}$ mass reaction rates $\left[\mathrm{kg} /\left(\mathrm{m}^{3} \cdot \mathrm{s}\right)\right]$. 
10. int TCDND_getTXC2RRml(double *scal, int $N_{\text {vars }}$, double *omega)

Returns molar reaction rates based on temperature $T$ and molar concentrations $\mathfrak{X}$ 's. If the nondimensional flag is ON (using TC_setNonDim) this function expects non-dimensional input and will provide non-dimensional output.

- scal - pointer to an array of $N_{\text {spec }}+1$ doubles $\left(T, \mathfrak{X}_{1}, \mathfrak{X}_{2}, \ldots, \mathfrak{X}_{N}\right)$, temperature $T[\mathrm{~K}]$, molar concentrations $\mathfrak{X}\left[\mathrm{kmol} / \mathrm{m}^{3}\right]$.

- $N_{\text {vars }}$ - no. of variables $=N_{\text {spec }}+1$

- omega - array of $N_{\mathrm{spec}}$ molar reaction rates $\left[\mathrm{kmol} /\left(\mathrm{m}^{3} \cdot \mathrm{s}\right)\right]$.

11. int TC_getTXC2RRml(double *scal, int $N_{\text {vars }}$, double *omega)

Returns molar reaction rates based on temperature $T$ and molar concentrations $\mathfrak{X}$ 's.

- scal - pointer to an array of $N_{\text {spec }}+1$ doubles $\left(T, \mathfrak{X}_{1}, \mathfrak{X}_{2}, \ldots, \mathfrak{X}_{N}\right)$, temperature $T[\mathrm{~K}]$, molar concentrations $\mathfrak{X}\left[\mathrm{kmol} / \mathrm{m}^{3}\right]$.

- $N_{\text {vars }}$ - no. of variables $=N_{\text {spec }}+1$

- omega - array of $N_{\mathrm{spec}}$ molar reaction rates $\left[\mathrm{kmol} /\left(\mathrm{m}^{3} \cdot \mathrm{s}\right)\right]$.

12. int TCDND_getTXC2RRms(double *scal, int $N_{\text {vars }}$, double *omega)

Returns non-dimensional mass reaction rates based on temperature $T$ and molar concentrations $\mathfrak{X}$ 's. If the non-dimensional flag is ON (using TC_setNonDim) this function expects non-dimensional input and will provide non-dimensional output.

- scal - pointer to an array of $N_{\text {spec }}+1$ doubles $\left(T, \mathfrak{X}_{1}, \mathfrak{X}_{2}, \ldots, \mathfrak{X}_{N}\right)$, temperature $T[\mathrm{~K}]$, molar concentrations $\mathfrak{X}\left[\mathrm{kmol} / \mathrm{m}^{3}\right]$.

- $N_{\text {vars }}$ - no. of variables $=N_{\text {spec }}+1$

- omega - array of $N_{\text {spec }}$ mass reaction rates $\left[\mathrm{kg} /\left(\mathrm{m}^{3} \cdot \mathrm{s}\right)\right]$.

13. int TC_getTXC2RRms(double *scal, int $N_{\text {vars }}$, double *omega)

Returns mass reaction rates based on temperature $T$ and molar concentrations $\mathfrak{X}$ 's.

- scal - pointer to an array of $N_{\text {spec }}+1$ doubles $\left(T, \mathfrak{X}_{1}, \mathfrak{X}_{2}, \ldots, \mathfrak{X}_{N}\right)$, temperature $T[\mathrm{~K}]$, molar concentrations $\mathfrak{X}\left[\mathrm{kmol} / \mathrm{m}^{3}\right]$.

- $N_{\text {vars }}$ - no. of variables $=N_{\text {spec }}+1$

- omega - array of $N_{\mathrm{spec}}$ mass reaction rates $\left[\mathrm{kg} /\left(\mathrm{m}^{3} \cdot \mathrm{s}\right)\right]$.

14. int TC_getRops(double $*$ scal, int $N_{\text {vars }}$, double $*$ datarop)

Returns rate-of-progress variables based on temperature $T$ and species mass fractions $Y$ 's.

- scal - pointer to an array of $N_{\text {spec }}+1$ doubles $\left(T, Y_{1}, Y_{2}, \ldots, Y_{N}\right)$, temperature $T[\mathrm{~K}]$, species mass fractions $Y[]$.

- $N_{\text {vars }}-$ no. of variables $=N_{\text {spec }}+1$

- datarop - array of $N_{\text {reac }}$ rate-of-progress variables $\left[\mathrm{kmol} /\left(\mathrm{m}^{3} \cdot \mathrm{s}\right)\right]$. 
15. int $\mathbf{T C}$ _getRfRb(double $*^{*}$ scal, int $N_{\text {vars }}$, double $*$ dataRfrb)

Returns rate-of-progress variables based on temperature $T$ and species mass fractions $Y$ 's.

- scal - pointer to an array of $N_{\text {spec }}+1$ doubles $\left(T, Y_{1}, Y_{2}, \ldots, Y_{N}\right)$, temperature $T[\mathrm{~K}]$, species mass fractions $Y$ [].

- $N_{\text {vars }}$ - no. of variables $=N_{\text {spec }}+1$

- dataRfrb - array of $N_{\text {reac }}$ forward rate-of-progress variables and $N_{\text {reac }}$ reverse rate-of-progress variables $\left[\mathrm{kmol} /\left(\mathrm{m}^{3} \cdot \mathrm{s}\right)\right]$.

\section{TC_spec.c}

\section{User functions:}

1. int TC_getNspec()

Returns number of species.

2. int TC_getNelem()

RReturns number of elements.

3. int $\mathbf{T C}$-getNvars()

Returns number of variables, currently no. of species plus one.

4. int TC_getSnames(int $N_{\text {spec }}$, char *snames)

Returns species names.

- $N_{\text {spec }}$ - no. of species, needs to match the library's internal value.

- snames - array of charecters, the allocation needs to be at least $N_{\text {spec }}$ *LENGTHOFSPECNAME. Currently LENGTHOFSPECNAME is set to 32. Name of species $i$ starts at position $i *$ LENGT HOF SPECNAME in the array.

\section{5. int TC_getSnameLen()}

Returns length of species names.

6. int $\mathbf{T C}$ _getSpos(const char *sname, const int slen)

Returns index of species sname.

- sname - name of species.

- slen - length of species name

7. int TC_getSmass(int $N_{\text {spec }}$, double $\left.* W i\right)$

Returns molar masses for all species.

- $N_{\text {spec }}$ - no. of species, needs to match the library's internal value.

- $W i$ - pointer to an array with molecular masses $[\mathrm{kg} / \mathrm{kmol}]$ for all species. The allocation size should be at least $N_{\text {spec }}$. 
1. int TCDND_getSrc(double *scal,int $N_{\text {vars }}$,double *omega)

Returns the source term for

$$
\frac{\partial T}{\partial t}=\omega_{0}, \frac{\partial Y_{i}}{\partial t}=\omega_{i}
$$

based on temperature $T$ and species mass fractions $Y$ 's. If the non-dimensional flag is ON (using TC_setNonDim) this function expects non-dimensional input and will provide non-dimensional output.

- scal - pointer to an pointer to an array of $N_{\text {spec }}+1$ doubles $\left(T, Y_{1}, Y_{2}, \ldots, Y_{N}\right)$, temperature $T$ [K], species mass fractions $Y$ [].

- $N_{\text {vars }}$ - no. of variables $=N_{\text {spec }}+1$

- omega : pointer to an array of doubles with the source terms for temperature and species mass fractions equations: omega $[0]:[\mathrm{K} / \mathrm{s}]$, omega $\left[1 \ldots N_{\mathrm{spec}}\right]:[1 / \mathrm{s}]$

2. int TC_getSrc(double *scal,int $N_{\text {vars }}$, double *omega)

Returns the source term for

$$
\frac{\partial T}{\partial t}=\omega_{0}, \frac{\partial Y_{i}}{\partial t}=\omega_{i}
$$

based on temperature $T$ and species mass fractions $Y$ 's.

- scal - pointer to an pointer to an of $N_{\text {spec }}+1$ doubles $\left(T, Y_{1}, Y_{2}, \ldots, Y_{N}\right)$, temperature $T$ [K], species mass fractions $Y$ [].

- $N_{\text {vars }}$ - no. of variables $=N_{\text {spec }}+1$

- omega : pointer to an array of doubles with the source terms for temperature and species mass fractions equations: omega $[0]:[\mathrm{K} / \mathrm{s}]$, omega $\left[1 \ldots N_{\text {spec }}\right]:[1 / \mathrm{s}]$

3. int TCDND_getSrcCons(double *scal,int $N_{\text {vars }}$, double *omega)

Returns the source term for

$$
\frac{\partial \rho}{\partial t}=\omega_{0}, \rho \frac{\partial Y_{i}}{\partial t}=\omega_{i}
$$

based on density $\rho$ and species mass fractions $Y^{\prime}$ 's. If the non-dimensional flag is ON (using TC_setNonDim) this function expects non-dimensional input and will provide non-dimensional output.

- scal - pointer to an array of $N_{\text {spec }}+1$ doubles $\left(\rho, Y_{1}, Y_{2}, \ldots, Y_{N}\right)$, density $\rho\left[\mathrm{kg} / \mathrm{m}^{3}\right]$, species mass fractions $Y$ [].

- $N_{\text {vars }}$ - no. of variables $=N_{\text {spec }}+1$

- omega : pointer to an array of doubles with the source terms for density and species mass fractions equations: omega $[0]:\left[\mathrm{kg} /\left(\mathrm{m}^{3} \cdot \mathrm{s}\right)\right]$, omega $\left[1 \ldots N_{\text {spec }}\right]:\left[\mathrm{kg} /\left(\mathrm{m}^{3} \cdot \mathrm{s}\right)\right]$ 
4. int $\mathbf{T C}$ _getSrcCons(double $*_{s c a l, \text { int }} N_{\text {vars }}$,double *omega)

Returns source term for

$$
\frac{\partial \rho}{\partial t}=\omega_{0}, \rho \frac{\partial Y_{i}}{\partial t}=\omega_{i}
$$

based on density $\rho$ and species mass fractions $Y$ 's.

- scal - pointer to an array of $N_{\text {spec }}+1$ doubles $\left(\rho, Y_{1}, Y_{2}, \ldots, Y_{N}\right)$, density $\rho\left[\mathrm{kg} / \mathrm{m}^{3}\right]$, species mass fractions $Y$ [].

- $N_{\text {vars }}$ - no. of variables $=N_{\text {spec }}+1$

- omega : pointer to an array with the source terms for density and species mass fractions equations: omega $[0]:\left[\mathrm{kg} /\left(\mathrm{m}^{3} \cdot \mathrm{s}\right)\right]$, omega $\left[1 \ldots N_{\mathrm{spec}}\right]:\left[\mathrm{kg} /\left(\mathrm{m}^{3} \cdot \mathrm{s}\right)\right]$

5. int TCDND_getJacTYNm1(double *scal, int $N_{\text {spec }}$, double *jac, unsigned int useJacAnl)

Computes Jacobian matrix for the system $\left(T, Y_{1}, Y_{2}, \ldots, Y_{N-1}\right)$ based on temperature $T$ and species mass fractions $Y$ 's using either analytical expressions of numerical derivatives. If the non-dimensional flag is ON (using TC_setNonDim) this function expects non-dimensional input and will provide nondimensional output.

- scal - pointer to an array of $N_{\text {spec }}+1$ doubles $\left(T, Y_{1}, Y_{2}, \ldots, Y_{N}\right)$, temperature $T[\mathrm{~K}]$, species mass fractions $Y[]$.

- $N_{\text {spec }}$ - no. of species $N_{\text {spec }}$

- jac - pointer to array of doubles with the Jacobian matrix $J$. Element $J_{i j}$ is stored at $j a c[j *$ $\left.N_{\text {spec }}+i\right]$.

- useJacAnl - flag for Jacobian type (1-analytical,other values-numerical)

6. int TC_getJacTYNm1(double $*_{s c a l}$, int $N_{\text {spec }}$, double $*_{\text {jac}}$, unsigned int useJacAnl)

Computes Jacobian matrix for the system $\left(T, Y_{1}, Y_{2}, \ldots, Y_{N-1}\right)$ based on temperature $T$ and species mass fractions $Y$ 's using either analytical expressions of numerical derivatives.

- scal - pointer to an pointer to an array of $N_{\text {spec }}+1$ doubles $\left(T, Y_{1}, Y_{2}, \ldots, Y_{N}\right)$, temperature $T$ [K], species mass fractions $Y$ [].

- $N_{\text {spec }}$ - no. of species $N_{\text {spec }}$

- jac - pointer to array of doubles with the Jacobian matrix $J$. Element $J_{i j}$ is stored at $j a c[j *$ $\left.N_{\text {spec }}+i\right]$.

- useJacAnl - flag for Jacobian type (1-analytical,other values-numerical)

7. int TCDND_getJacTYNm1anl(double $*_{s c a l, \text { int }} N_{\mathrm{spec}}$, double $*$ jac)

Computes Jacobian matrix for the system $\left(T, Y_{1}, Y_{2}, \ldots, Y_{N-1}\right)$ based on temperature $T$ and species mass fractions $Y$ 's using either analytical expressions. If the non-dimensional flag is ON (using TC_setNonDim) this function expects non-dimensional input and will provide non-dimensional output.

- scal - pointer to an array of $N_{\text {spec }}+1$ doubles $\left(T, Y_{1}, Y_{2}, \ldots, Y_{N}\right)$, temperature $T[\mathrm{~K}]$, species mass fractions $Y$ []. 
- $N_{\text {spec }}$ - no. of species $N_{\text {spec }}$

- jac - pointer to array of doubles with the Jacobian matrix $J$. Element $J_{i j}$ is stored at $j a c[j *$ $\left.N_{\text {spec }}+i\right]$.

8. int TC_getJacTYNm1anl(double $*_{s c a l}$, int $N_{\text {spec }}$, double $*$ jac)

Computes Jacobian matrix for the system $\left(T, Y_{1}, Y_{2}, \ldots, Y_{N-1}\right)$ based on temperature $T$ and species mass fractions $Y$ 's using either analytical expressions.

- scal - pointer to an array of $N_{\text {spec }}+1$ doubles $\left(T, Y_{1}, Y_{2}, \ldots, Y_{N}\right)$, temperature $T[\mathrm{~K}]$, species mass fractions $Y$ [].

- $N_{\text {spec }}$ - no. of species $N_{\text {spec }}$

- jac - pointer to array of doubles with the Jacobian matrix $J$. Element $J_{i j}$ is stored at $j a c[j *$ $\left.N_{\text {spec }}+i\right]$.

9. int TCDND_getJacTYN(double $*$ scal, int $N_{\mathrm{spec}}$, double $*$ jac, unsigned int useJacAnl)

Computes Jacobian matrix for the system $\left(T, Y_{1}, Y_{2}, \ldots, Y_{N}\right)$ based on temperature $T$ and species mass fractions $Y$ 's using either analytical expressions of numerical derivatives. If the non-dimensional flag is ON (using TC_setNonDim) this function expects non-dimensional input and will provide nondimensional output.

- scal - pointer to an array of $N_{\text {spec }}+1$ doubles $\left(T, Y_{1}, Y_{2}, \ldots, Y_{N}\right)$, temperature $T[\mathrm{~K}]$, species mass fractions $Y$ [].

- $N_{\text {spec }}$ - no. of species $N_{\text {spec }}$

- jac - pointer to array of doubles with the Jacobian matrix $J$. Element $J_{i j}$ is stored at $j a c[j *$ $\left.\left(N_{\text {spec }}+1\right)+i\right]$.

- useJacAnl - flag for Jacobian type (1-analytical,other values-numerical)

10. int TC_getJacTYN(double *scal, int $N_{\mathrm{spec}}$, double $*_{\mathrm{j}}$ ac, unsigned int useJacAnl)

Computes Jacobian matrix for the system $\left(T, Y_{1}, Y_{2}, \ldots, Y_{N}\right)$ based on temperature $T$ and species mass fractions $Y$ 's using either analytical expressions of numerical derivatives.

- scal - pointer to an array of $N_{\text {spec }}+1$ doubles $\left(T, Y_{1}, Y_{2}, \ldots, Y_{N}\right)$, temperature $T[\mathrm{~K}]$, species mass fractions $Y[]$.

- $N_{\text {spec }}$ - no. of species $N_{\text {spec }}$

- jac - pointer to array of doubles with the Jacobian matrix $J$. Element $J_{i j}$ is stored at $j a c[j *$ $\left.\left(N_{\text {spec }}+1\right)+i\right]$.

- useJacAnl - flag for Jacobian type (1-analytical,other values-numerical)

11. int TCDND_getJacTYNanl(double *scal, int $N_{\text {spec }}$, double *jac)

Computes Jacobian matrix for the system $\left(T, Y_{1}, Y_{2}, \ldots, Y_{N}\right)$ based on temperature $T$ and species mass fractions $Y$ 's using either analytical expressions. If the non-dimensional flag is ON (using TC_setNonDim) this function expects non-dimensional input and will provide non-dimensional output. 
- scal - pointer to an array of $N_{\text {spec }}+1$ doubles $\left(T, Y_{1}, Y_{2}, \ldots, Y_{N}\right)$, temperature $T[\mathrm{~K}]$, species mass fractions $Y[]$.

- $N_{\text {spec }}$ - no. of species $N_{\text {spec }}$

- jac - pointer to array of doubles with the Jacobian matrix $J$. Element $J_{i j}$ is stored at $j a c[j *$ $\left.\left(N_{\text {spec }}+1\right)+i\right]$.

12. int TC_getJacTYNanl(double $*_{s c a l}$, int $N_{\text {spec }}$, double $*_{\text {jac }}$ )

Computes Jacobian matrix for the system $\left(T, Y_{1}, Y_{2}, \ldots, Y_{N}\right)$ based on temperature $T$ and species mass fractions $Y$ 's using either analytical expressions.

- scal - pointer to an array of $N_{\text {spec }}+1$ doubles $\left(T, Y_{1}, Y_{2}, \ldots, Y_{N}\right)$, temperature $T[\mathrm{~K}]$, species mass fractions $Y$ [].

- $N_{\text {spec }}$ - no. of species $N_{\text {spec }}$

- jac - pointer to array of doubles with the Jacobian matrix $J$. Element $J_{i j}$ is stored at $j a c[j *$ $\left.\left(N_{\text {spec }}+1\right)+i\right]$.

13. int TC_getJacRPTYN(double *scal, int $N_{\text {spec }}$, double *jac, unsigned int useJacAnl)

Computes Jacobian matrix for the system $\left(\rho, P, T, Y_{1}, Y_{2}, \ldots, Y_{N}\right)$ based on temperature $T$ and species mass fractions $Y$ 's using either analytical expressions of numerical derivatives.

- scal - pointer to an array of $N_{\text {spec }}+1$ doubles $\left(T, Y_{1}, Y_{2}, \ldots, Y_{N}\right)$, temperature $T[\mathrm{~K}]$, species mass fractions $Y$ [].

- $N_{\text {spec }}$ - no. of species $N_{\text {spec }}$

- jac - pointer to array of doubles with the Jacobian matrix $J$. Element $J_{i j}$ is stored at $j a c[j *$ $\left.\left(N_{\text {spec }}+3\right)+i\right]$.

- useJacAnl : flag for Jacobian type (1-analytical,other values-numerical).

14. int TC_getJacRPTYNanl(double *scal, int $N_{\text {spec }}$, double $*$ jac)

Computes the Jacobian matrix for the system $\left(\rho, P, T, Y_{1}, Y_{2}, \ldots, Y_{N}\right)$ based on temperature $T$ and species mass fractions $Y$ 's using analytical expressions.

- scal - pointer to an array of $N_{\mathrm{spec}}+1$ doubles $\left(T, Y_{1}, Y_{2}, \ldots, Y_{N}\right)$, temperature $T[\mathrm{~K}]$, species mass fractions $Y$ [].

- $N_{\text {spec }}$ - no. of species $N_{\text {spec }}$

- jac - pointer to array of doubles with the Jacobian matrix $J$. Element $J_{i j}$ is stored at $j a c[j *$ $\left.\left(N_{\mathrm{spec}}+3\right)+i\right]$.

15. int TC_getJacRPTYNnum(double $*_{s c a l}$, int $N_{\text {spec }}$, double $*$ jac)

Computes the Jacobian matrix for the system $\left(\rho, P, T, Y_{1}, Y_{2}, \ldots, Y_{N}\right)$ based on temperature $T$ and species mass fractions $Y$ 's using numerical derivatives.

- scal - pointer to an array of $N_{\text {spec }}+1$ doubles $\left(T, Y_{1}, Y_{2}, \ldots, Y_{N}\right)$, temperature $T[\mathrm{~K}]$, species mass fractions $Y$ [].

- $N_{\text {spec }}$ - no. of species $N_{\text {spec }}$

- jac - pointer to array of doubles with the Jacobian matrix $J$. Element $J_{i j}$ is stored at $j a c[j *$ $\left.\left(N_{\text {spec }}+3\right)+i\right]$. 
1. int TCDND_getRhoMixMs(double *scal,int $N_{\text {vars }}$, double *rhomix)

Computes density based on temperature $T$ and species mass fractions $Y$ 's using the equation of state. If the non-dimensional flag is ON (using TC_setNonDim) this function expects non-dimensional input and will provide non-dimensional output.

- scal - pointer to an array of $N_{\text {spec }}+1$ doubles $\left(T, Y_{1}, Y_{2}, \ldots, Y_{N}\right)$, temperature $T[\mathrm{~K}]$, species mass fractions $Y$ [].

- $N_{\text {vars }}$ - no. of variables $=N_{\text {spec }}+1$

- rhomix - pointer to mixture density $\left[\mathrm{kg} / \mathrm{m}^{3}\right]$

2. int TC_getRhoMixMs(double $*$ scal, int $N_{\text {vars }}$,double *rhomix)

Computes density based on temperature $T$ and species mass fractions $Y$ 's using the equation of state.

- scal - pointer to an array of $N_{\text {spec }}+1$ doubles $\left(T, Y_{1}, Y_{2}, \ldots, Y_{N}\right)$, temperature $T[\mathrm{~K}]$, species mass fractions $Y[]$.

- $N_{\text {vars }}$ - no. of variables $=N_{\text {spec }}+1$

- rhomix - pointer to mixture density $\left[\mathrm{kg} / \mathrm{m}^{3}\right]$

3. int TCDND_getRhoMixMl(double *scal,int $N_{\text {vars }}$, double *rhomix)

Computes density based on temperature $T$ and species mole fractions $X$ 's using the equation of state. If the non-dimensional flag is ON (using TC_setNonDim) this function expects non-dimensional input and will provide non-dimensional output.

- scal - pointer to an array of $N_{\text {spec }}+1$ doubles $\left(T, X_{1}, X_{2}, \ldots, X_{N}\right)$, temperature $T[\mathrm{~K}]$, species mole fractions $X[]$.

- $N_{\text {vars }}$ - no. of variables $=N_{\text {spec }}+1$

- rhomix - pointer to mixture density $\left[\mathrm{kg} / \mathrm{m}^{3}\right]$

4. int TC_getRhoMixMl(double $*$ scal,int $N_{\text {vars }}$, double *rhomix)

Computes density based on temperature $T$ and species mole fractions $X$ 's using the equation of state.

- scal - pointer to an array of $N_{\text {spec }}+1$ doubles $\left(T, X_{1}, X_{2}, \ldots, X_{N}\right)$, temperature $T[\mathrm{~K}]$, species mole fractions $X[]$.

- $N_{\text {vars }}$ - no. of variables $=N_{\text {spec }}+1$

- rhomix - pointer to mixture density $\left[\mathrm{kg} / \mathrm{m}^{3}\right]$

5. int TCDND_getTMixMs(double *scal,int $N_{\text {vars }}$, double *Tmix)

Computes temperature based on density $\rho$ and species mass fractions $Y$ 's using the equation of state. If the non-dimensional flag is ON (using TC_setNonDim) this function expects non-dimensional input and will provide non-dimensional output.

- scal - pointer to an array of $N_{\text {spec }}+1$ doubles $\left(\rho, Y_{1}, Y_{2}, \ldots, Y_{N}\right)$, density $\rho\left[\mathrm{kg} / \mathrm{m}^{3}\right]$, species mass fractions $Y$ []. 
- $N_{\text {vars }}$ - no. of variables $=N_{\text {spec }}+1$

- Tmix - pointer to temperature $[\mathrm{K}]$

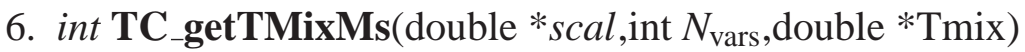

Computes temperature based on density $\rho$ and species mass fractions $Y$ 's using the equation of state.

- scal - pointer to an array of $N_{\text {spec }}+1$ doubles $\left(\rho, Y_{1}, Y_{2}, \ldots, Y_{N}\right)$, density $\rho\left[\mathrm{kg} / \mathrm{m}^{3}\right]$, species mass fractions $Y$ [].

- $N_{\text {vars }}$ - no. of variables $=N_{\text {spec }}+1$

- Tmix - pointer to temperature [K]

7. int TCDND_getTMixMl(double $*$ scal,int $N_{\text {vars }}$,double $*$ Tmix)

Computes temperature based on density $\rho$ and species mole fractions $X$ 's using the equation of state. If the non-dimensional flag is ON (using TC_setNonDim) this function expects non-dimensional input and will provide non-dimensional output.

- scal - pointer to an array of $N_{\text {spec }}+1$ doubles $\left(\rho, X_{1}, X_{2}, \ldots, X_{N}\right)$, density $\rho\left[\mathrm{kg} / \mathrm{m}^{3}\right]$, species mole fractions $X[]$.

- $N_{\text {vars }}$ - no. of variables $=N_{\text {spec }}+1$

- Tmix - pointer to temperature $[\mathrm{K}]$

8. int TC_getTMixMl(double $*$ scal,int $N_{\text {vars }}$, double $*$ Tmix)

Computes temperature based on density $\rho$ and species mole fractions $X$ 's using the equation of state.

- scal - pointer to an array of $N_{\text {spec }}+1$ doubles $\left(\rho, X_{1}, X_{2}, \ldots, X_{N}\right)$, density $\rho\left[\mathrm{kg} / \mathrm{m}^{3}\right]$, species mole fractions $X[]$.

- $N_{\text {vars }}$ - no. of variables $=N_{\text {spec }}+1$

- Tmix - pointer to temperature [K]

9. int TCDND_getMs2CpMixMs(double *scal,int $N_{\text {vars }}$, double *cpmix)

Computes mixture specific heat at constant pressure based on temperature $T$ and species mass fractions $Y$ 's. If the non-dimensional flag is ON (using TC setNonDim) this function expects nondimensional input and will provide non-dimensional output.

- scal - pointer to an array of $N_{\text {spec }}+1$ doubles $\left(T, Y_{1}, Y_{2}, \ldots, Y_{N}\right)$, temperature $T[\mathrm{~K}]$, species mass fractions $Y$ [].

- $N_{\text {vars }}$ - no. of variables $=N_{\text {spec }}+1$

- cpmix - pointer to mixture specific heat at constant pressure $[\mathrm{J} /(\mathrm{kg} \cdot \mathrm{K})]$

10. int TC_getMs2CpMixMs(double $*_{\text {scal, int }} N_{\text {vars }}$, double $*$ cpmix)

Computes mixture specific heat at constant pressure based on temperature $T$ and species mass fractions $Y$ 's.

- scal -pointer to an array of $N_{\mathrm{spec}}+1$ doubles $\left(T, Y_{1}, Y_{2}, \ldots, Y_{N}\right)$, temperature $T$ [K], species mass fractions $Y$ []. 
- $N_{\text {vars }}$ - no. of variables $=N_{\text {spec }}+1$

- cpmix - pointer to mixture specific heat at constant pressure $[\mathrm{J} /(\mathrm{kg} \cdot \mathrm{K})]$

11. int TCDND_getMs2CvMixMs(double $*$ scal,int $N_{\text {vars }}$, double $*$ cvmix)

Computes mixture specific heat at constant volume based on temperature $T$ and species mass fractions $Y$ 's. If the non-dimensional flag is $\mathrm{ON}$ (using TC_setNonDim) this function expects nondimensional input and will provide non-dimensional output.

- scal - pointer to an array of $N_{\text {spec }}+1$ doubles $\left(T, Y_{1}, Y_{2}, \ldots, Y_{N}\right)$, temperature $T[\mathrm{~K}]$, species mass fractions $Y$ [].

- $N_{\text {vars }}$ - no. of variables $=N_{\text {spec }}+1$

- cvmix - pointer to mixture specific heat at constant volume $[\mathrm{J} /(\mathrm{kg} \cdot \mathrm{K})]$

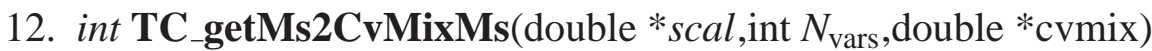

Computes mixture specific heat at constant volume based on temperature $T$ and species mass fractions $Y$ 's.

- scal - pointer to an array of $N_{\text {spec }}+1$ doubles $\left(T, Y_{1}, Y_{2}, \ldots, Y_{N}\right)$, temperature $T[\mathrm{~K}]$, species mass fractions $Y$ [].

- $N_{\text {vars }}$ - no. of variables $=N_{\text {spec }}+1$

- cvmix - pointer to mixture specific heat at constant volume $[\mathrm{J} /(\mathrm{kg} \cdot \mathrm{K})]$

13. int TCDND_getMI2CpMixMl(double $*_{\text {scal, int }} N_{\text {vars }}$, double $*$ cvmix)

Computes mixture heat capacity at constant pressure based on temperature $T$ and species mole fractions $X$ 's. If the non-dimensional flag is ON (using TC_setNonDim) this function expects nondimensional input and will provide non-dimensional output.

- scal - pointer to an array of $N_{\text {spec }}+1$ doubles $\left(T, X_{1}, X_{2}, \ldots, X_{N}\right)$, temperature $T[\mathrm{~K}]$, species mole fractions $X[]$.

- $N_{\text {vars }}$ - no. of variables $=N_{\text {spec }}+1$

- cvmix - pointer to mixture heat capacity at constant volume $[\mathrm{J} /(\mathrm{kmol} \cdot \mathrm{K})]$

14. int TC_getMl2CpMixMl(double $*$ scal,int $N_{\text {vars }}$, double $*$ cvmix)

Computes mixture heat capacity at constant pressure based on temperature $T$ and species mole fractions $X$ 's.

- scal - pointer to an array of $N_{\text {spec }}+1$ doubles $\left(T, X_{1}, X_{2}, \ldots, X_{N}\right)$, temperature $T[\mathrm{~K}]$, species mole fractions $X[]$.

- $N_{\text {vars }}$ - no. of variables $=N_{\text {spec }}+1$

- cvmix - pointer to mixture heat capacity at constant volume $[\mathrm{J} /(\mathrm{kmol} \cdot \mathrm{K})]$

15. int TCDND_getCpSpecMs(double $t$, int $N_{\text {spec }}$,double $*$ cpi)

Computes species specific heat at constant pressure based on temperature $T$. If the non-dimensional flag is ON (using TC_setNonDim) this function expects non-dimensional input and will provide non-dimensional output. 
- $t$ - temperature $T[\mathrm{~K}]$

- $N_{\text {spec }}-$ no. of species

- cpi - array with species specific heats at constant pressure $[\mathrm{J} /(\mathrm{kg} \cdot \mathrm{K})]$

16. int TC_getCpSpecMs(double $t$, int $N_{\text {spec }}$, double $*$ cpi)

Computes species specific heat at constant pressure based on temperature $T$.

- $t$ - temperature $T[\mathrm{~K}]$

- $N_{\text {spec }}-$ no. of species

- cpi - array with species specific heats at constant pressure $[\mathrm{J} /(\mathrm{kg} \cdot \mathrm{K})]$

17. int TCDND_getCpSpecMl(double $t$, int $N_{\text {spec }}$, double $*$ cpi)

Computes species heat capacities at constant pressure based on temperature $T$. If the non-dimensional flag is ON (using TC_setNonDim) this function expects non-dimensional input and will provide nondimensional output.

- $t$ - temperature $T[\mathrm{~K}]$

- $N_{\text {spec }}-$ no. of species

- cpi - array with species heat capacities at constant pressure $[\mathrm{J} /(\mathrm{kmol} \cdot \mathrm{K})]$

18. int TC_getCpSpecMl(double $t$, int $N_{\text {spec }}$, double $*$ cpi)

Computes species heat capacities at constant pressure based on temperature.

- $t$ - temperature $T[\mathrm{~K}] Y[]$.

- $N_{\text {spec }}-$ no. of species

- cpi - array with species heat capacities at constant pressure $[\mathrm{J} /(\mathrm{kmol} \cdot \mathrm{K})]$

19. int TCDND_getMs2HmixMs(double $*_{s c a l, \text { int }} N_{\text {vars }}$, double $\left.* h m i x\right)$

Computes mixture specific enthalpy based on temperature and species mass fractions. If the nondimensional flag is ON (using TC_setNonDim) this function expects non-dimensional input and will provide non-dimensional output.

- scal - pointer to an array of $N_{\text {spec }}+1$ doubles $\left(T, Y_{1}, Y_{2}, \ldots, Y_{N}\right)$, temperature $T[\mathrm{~K}]$, species mass fractions $Y$ [].

- $N_{\text {vars }}-$ no. of variables $=N_{\text {spec }}+1$

- hmix - pointer to mixture specific enthalpy $[\mathrm{J} / \mathrm{kg}]$.

20. int TC_getMs2HmixMs(double *scal,int $N_{\text {vars }}$, double *hmix)

Computes mixture specific enthalpy based on temperature and species mass fractions.

- scal - pointer to an array of $N_{\text {spec }}+1$ doubles $\left(T, Y_{1}, Y_{2}, \ldots, Y_{N}\right)$, temperature $T[\mathrm{~K}]$, species mass fractions $Y$ [].

- $N_{\text {vars }}$ - no. of variables $=N_{\text {spec }}+1$

- hmix - pointer to mixture specific enthalpy $[\mathrm{J} / \mathrm{kg}]$. 


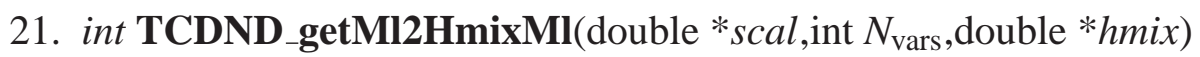

Computes mixture molar enthalpy based on temperature and species mole fractions. If the nondimensional flag is ON (using TC_setNonDim) this function expects non-dimensional input and will provide non-dimensional output.

- scal - pointer to an array of $N_{\text {spec }}+1$ doubles $\left(T, X_{1}, X_{2}, \ldots, X_{N}\right)$, temperature $T[\mathrm{~K}]$, species mole fractions $X[]$.

- $N_{\text {vars }}$ - no. of variables $=N_{\text {spec }}+1$

- hmix - pointer to mixture molar enthalpy [J/kmol].

22. int TC_getMl2HmixMl(double $*$ scal, int $N_{\text {vars }}$, double $*$ hmix $)$

Computes mixture molar enthalpy based on temperature $T$ and species mole fractions $X$ 's.

- scal - pointer to an array of $N_{\text {spec }}+1$ doubles $\left(T, X_{1}, X_{2}, \ldots, X_{N}\right)$, temperature $T[\mathrm{~K}]$, species mole fractions $X[]$.

- $N_{\text {vars }}$ - no. of variables $=N_{\text {spec }}+1$

- hmix - pointer to mixture molar enthalpy [J/kmol].

23. int TCDND_getHspecMs(double $t$, int $N_{\text {spec }}$, double $* h i$ )

Computes species specific enthalpies based on temperature $T$. If the non-dimensional flag is ON (using TC_setNonDim) this function expects non-dimensional input and will provide non-dimensional output.

- $t$ - temperature $T[\mathrm{~K}]$

- $N_{\text {spec }}$ - no. of species

- $h i$ - array of $N_{\text {spec }}$ doubles with species specific enthalpies $[\mathrm{J} / \mathrm{kg}]$

24. int $\mathbf{T C}$ _getHspecMs(double $t$, int $N_{\text {spec }}$, double $* h i$ )

Computes species specific enthalpies based on temperature $T$.

- $t$ - temperature $T[\mathrm{~K}]$

- $N_{\text {spec }}$ - no. of species

- $h i$ - array of $N_{\text {spec }}$ doubles with species specific enthalpies [J/kg]

25. int TCDND_getHspecMl(double $t$, int $N_{\text {spec }}$, double *hi)

Computes species molar enthalpies based on temperature $T$. If the non-dimensional flag is ON (using TC_setNonDim) this function expects non-dimensional input and will provide non-dimensional output.

- $t$ - temperature $T[\mathrm{~K}]$

- $N_{\text {spec }}$ - no. of species

- $h i$ - array of $N_{\text {spec }}$ doubles with species molar enthalpies [J/kmol]

26. int TC_getHspecMl(double $t$, int $N_{\text {spec }}$, double *hi)

Computes species molar enthalpies based on temperature $T$. 
- $t$ - temperature $T[\mathrm{~K}]$

- $N_{\text {spec }}$ - no. of species

- $h i$ - array of $N_{\text {spec }}$ doubles with species molar enthalpies [J/kmol] 


\section{Chapter 7}

\section{Nomenclature}

- Temperature : $T-[K]$.

- Density : $\rho-\left[\frac{\mathrm{kg}}{\mathrm{m}^{3}}\right]$.

- Thermodynamic pressure : $P$ - $\left[\frac{\mathrm{kg}}{\mathrm{m} \cdot \mathrm{s}}\right]$.

- Mass and mole fraction of species $k: Y_{k}$ and $X_{k}$, respectively - [].

- Molar concentration of species $k: \mathfrak{X}_{k}$, respectively - $\left[\frac{\mathrm{kmol}}{\mathrm{m}^{3}}\right]$.

- Molecular weight of species $k$ and of the mixture: $W_{k}$ and $\bar{W}$, respectively - $\left[\frac{g}{\mathrm{~mol}}\right]=\left[\frac{\mathrm{kg}}{\mathrm{kmol}}\right]$.

- Universal gas constant, $\mathfrak{R}=8.314472 \times 10^{3}\left[\frac{\mathrm{J}}{\mathrm{kmol} \cdot \mathrm{K}}\right]=1.98721\left[\frac{\mathrm{cal}}{\mathrm{mol} \cdot \mathrm{K}}\right]$.

- Molar heat capacity at constant pressure (species $k$ and mixture): $C_{p, k}$ and $C_{p}$, respectively - $\left[\frac{\mathrm{J}}{\mathrm{kmol} \cdot K}\right]$.

- Specific heat capacity at constant pressure (species $k$ and mixture): $c_{p, k}$ and $c_{p}$, respectively - $\left[\frac{\mathrm{J}}{\mathrm{kg} \cdot K}\right]$.

- Molar enthalpy (species $k$ and mixture): $H_{k}$ and $H$, respectively - $\left[\frac{J}{k m o l}\right]$.

- Specific enthalpy (species $k$ and mixture): $h_{k}$ and $h$, respectively - $\left[\frac{J}{k g}\right]$.

- Mass reaction rate of species $k: \dot{\omega}_{k}-\left[\frac{\mathrm{kg}}{\mathrm{m}^{3} \cdot s}\right]$.

- Molar reaction rate of species $k: \dot{\omega}_{k}-\left[\frac{\mathrm{kmol}}{\mathrm{m}^{3} \cdot \mathrm{s}}\right]$.

\section{Non-dimensional values}

- Temperature : $T^{*}=T / T_{\text {ref }}$.

- Density : $\rho^{*}=\rho / \rho_{\text {ref }}$. 
- Molar concentration of species $k: \mathfrak{X}_{k}^{*}=\mathfrak{X}_{k} \cdot W_{\text {ref }} / \rho_{\text {ref }}$.

- Molecular weight of species $k$ and of the mixture: $W_{k}$ and $\bar{W}$, respectively - $\left[\frac{g}{m o l}\right]=\left[\frac{\mathrm{kg}}{\mathrm{kmol}}\right]$.

- Molar heat capacity at constant pressure (species $k$ and mixture): $C_{p, k}^{*}=C_{p, k} \cdot W_{\text {ref }} / c_{p_{\text {ref }}}$ and $C_{p}^{*}=$ $C_{p} \cdot W_{\text {ref }} / c_{p_{\text {ref }}}$, respectively.

- Specific heat capacity at constant pressure (species $k$ and mixture): $c_{p, k}^{*}=c_{p, k} / c_{p_{\text {ref }}}$ and $c_{p}^{*}=$ $c_{p} / c_{p_{\text {ref }}}$, respectively.

- Molar enthalpy (species $k$ and mixture): $H_{k}^{*}=H_{k} \cdot W_{\text {ref }} / h_{\text {ref }}$ and $H^{*}=H \cdot W_{\text {ref }} / h_{\text {ref }}$, respectively.

- Specific enthalpy (species $k$ and mixture): $h_{k}^{*}=h_{k} / h_{\text {ref }}$ and $h^{*}=h / h_{\text {ref }}$, respectively.

- Mass reaction rate of species $k: \dot{\omega}_{k}^{*}=\dot{\omega}_{k} \cdot t i m_{\mathrm{ref}} / \rho_{\text {ref }}$

- Molar reaction rate of species $k: \dot{\omega}_{k}^{*}=\dot{\omega}_{k} \cdot W_{\text {ref }} t i m_{\text {ref }} / \rho_{\text {ref }}$. 


\section{References}

[1] P. N. Brown, G. D. Byrne, and A. C. Hindmarsh. VODE: A Variable Coefficient ODE Solver. SIAM J. Sci. Stat. Comput., 10:1038-1051, 1989.

[2] S. D. Cohen and A. C. Hindmarsh. CVODE, a Stiff/Nonstiff ODE Solver in C. Comput. Phys., 10(2):138-143, 1996.

[3] H.J. Curran, P. Gaffuri, W.J. Pitz, and C.K. Westbrook. A comprehensive modeling study of iso-octane oxidation. Combust. Flame, 129:253-280, 2002.

[4] H.J. Curran, W.J. Pitz, C.K. Westbrook, C.V. Callahan, and F.L. Dryer. Oxidation of automotive primary reference fuels at elevated pressures. Proc. Comb. Inst., 27:379-387, 1998.

[5] W. J. Pitz M. Mehl, H. J. Curran and C. K. Westbrook. Chemical kinetic modeling of component mixtures relevant to gasoline. European Combustion Meeting, 2009.

[6] B. J. McBride, S. Gordon, and M. A. Reno. Coefficients for Calculating Thermodynamic and Transport Properties of Individual Species. Technical Report NASA TM-4513, NASA, 1993.

[7] G.P. Smith, D.M. Golden, M. Frenklach, N.W. Moriarty, B. Eiteneer, M. Goldenberg, C.T. Bowman, R.K. Hanson, S. Song, W.C. Gardiner Jr., V.V. Lissianski, and Q. Zhiwei. www.me.berkeley.edu/gri_mech/. 


$\begin{array}{lll}1 & \text { MS 9051 } & \text { Habib Najm, } 8351 \text { (electronic copy) } \\ 1 & \text { MS 9051 } & \text { Bert Debusschere, 8351 (electronic copy) } \\ 1 & \text { MS 9155 } & \text { Jerry McNeish, 8954 (electronic copy) } \\ 1 & \text { MS 9051 } & \text { Damian Rouson, 8351 (electronic copy) } \\ 1 & \text { MS 9051 } & \text { Cosmin Safta, 8954 (electronic copy) } \\ 1 & \text { MS 9151 } & \text { Jim Costa, 8950 (electronic copy) } \\ 1 & \text { MS 9054 } & \text { Andrew Mcllroy, 8350 (electronic copy) } \\ 1 & \text { MS } 0899 & \text { Technical Library, 9536 (electronic copy) }\end{array}$




\section{(1) Sandia National Laboratories}

